\title{
BRAIN PROJECTIONS FROM THE MEDULLARY DORSAL RETICULAR NUCLEUS: AN ANTEROGRADE AND RETROGRADE TRACING STUDY IN THE RAT
}

\section{H. LEITE-ALMEIDA, A. VALLE-FERNANDES AND A. ALMEIDA*}

Life and Health Sciences Research Institute, School of Health Sciences, University of Minho, CP-II, Piso 3, Campus de Gualtar, 4710057 Braga, Portugal

Abstract-In the last 15 years a role has been ascribed for the medullary dorsal reticular nucleus as a supraspinal pain modulating area. The medullary dorsal reticular nucleus is reciprocally connected with the spinal dorsal horn, is populated mainly by nociceptive neurons and regulates spinal nociceptive processing. Here we analyze the distribution of brain projections from the medullary dorsal reticular nucleus using the iontophoretic administration of the anterograde tracer biotinylateddextran amine and the retrograde tracer cholera toxin subunit $B$.

Fibers and terminal boutons labeled from the medullary dorsal reticular nucleus were located predominately in the brainstem, although extending also to the forebrain. In the medulla oblongata, anterograde labeling was observed in the orofacial motor nuclei, inferior olive, caudal ventrolateral medulla, rostral ventromedial medulla, nucleus tractus solitarius and most of the reticular formation. Labeling at the pons-cerebellum level was present in the locus coeruleus, A5 and A7 noradrenergic cell groups, parabrachial and deep cerebellar nuclei, whereas in the mesencephalon it was located in the periaqueductal gray matter, deep mesencephalic, oculomotor and anterior pretectal nuclei, and substantia nigra. In the diencephalon, fibers and terminal boutons were found mainly in the parafascicular, ventromedial, and posterior thalamic nuclei and in the arcuate, lateral, posterior, peri- and paraventricular hypothalamic areas. Telencephalic labeling was consistent but less intense and concentrated in the septal nuclei, globus pallidus and amygdala.

The well-known role of the medullary dorsal reticular nucleus in nociception and its pattern of brain projections in rats suggests that the nucleus is possibly implicated in the modulation of: (i) the ascending nociceptive transmission involved in the motivational-affective dimension of pain; (ii) the endogenous supraspinal pain control system centered in the periaqueductal gray matter-rostral ventromedial medulla-spinal cord circuitry; (iii) the motor reactions associated with pain. (C) 2006 Published by Elsevier Ltd on behalf of IBRO.

Key words: supraspinal circuitry, medullary reticular formation, pain pathways, cholera toxin subunit $B$, biotinylated dextran, brain efferents.

${ }^{*}$ Corresponding author. Tel: +351-253-604808; fax: +351-253-604809. E-mail address: aalmeida@ecsaude.uminho.pt (A. Almeida).

Abbreviations: (The nomenclature and abbreviations used to designate brain nuclei and fiber tracts are in accordance with those used by Paxinos and Watson (1998) or result from a simplification of it, except for a few exceptions assigned with (*).) ABC, avidin-biotin complex; ac/aca, anterior commissure/anterior part; $\mathrm{AH}$, anterior hypothalamic area; Amy, amygdaloid nuclei; AP, area postrema; APT, anterior pretectal nucleus; Aq, Sylvius aqueduct; Arc, arcuate hypothalamic nuclei; A5/A7, A5/A7 noradrenaline cells; BDA, biotinylated dextran; BLA, basolateral amygdadoi:10.1016/j neuroscience.2006.02.022

A growing amount of evidence indicates that supraspinal pain control centers involved in the modulation of spinal

loid nucleus, anterior part; BST, bed nucleus of the stria terminalis; $\mathrm{cc}$, central canal; $\mathrm{Ce}$, central amygdaloid nuclei; $\mathrm{CL}$, centrolateral thalamic nucleus; $\mathrm{CM}$, central medial thalamic nucleus; $\mathrm{CnF}$, cuneiform nucleus; $\mathrm{cp}$, cerebral peduncle basal part; $\mathrm{CPu}$, caudate putamen; CTb, cholera toxin subunit B; $\mathrm{Cu}$, cuneate nucleus; DK, nucleus of Darkschewitsch; DPAG, dorsal periaqueductal gray; DPGi, dorsal paragigantocellular nucleus; DpMe, deep mesencephalic nucleus; DR, dorsal raphe; DRt, * dorsal reticular nucleus; DTg, dorsal tegmental nucleus; ECu, external cuneate nucleus; En, endopiriform nuclei; Eth, ethmoid thalamic nucleus; $f$, fornix; $F$, nucleus of the fields of Forel; fr, fasciculus retroflexus; $G i$ gigantocellular reticular nucleus; GiA, gigantocellular reticular nucleus alpha part; GiV, gigantocellular reticular nucleus ventral part; GP, globus pallidus; $\mathrm{Gr}$, gracile nucleus; HDB, nucleus of the horizontal limb of the diagonal band; ic, internal capsule; IC, inferior colliculus; ICj, islands of Calleja; icp, inferior cerebellar peduncle; IG, indusium griseum; IMLF, interstitial nucleus of the medial longitudinal fasciculus; Int, interposed cerebellar nucleus; IO/IOM, inferior olive/medial nucleus; IP, interpeduncular nuclei; IRt, intermediate reticular nucleus; KF, Kölliker-Fuse nucleus; Lat, lateral (dentate) cerebellar nucleus; LC, locus coeruleus; Ifp, longitudinal fasciculus of the pons; LGP, lateral globus pallidus; LH, latera hypothalamus; II, lateral lemniscus; LL, nuclei of the lateral lemniscus; LM, lateral mammillary nucleus; LPB, lateral parabrachial nuclei; LPGi, lateral paragigantocellular nucleus; LPO, lateral preoptic nuclei; LRt, lateral reticular nucleus; LS/LSI, lateral septal nuclei/intermediate part; LV, lateral ventricle; MCPO, magnocellular preoptic nucleus; Me, medial amygdaloid nuclei; Me5, mesencephalic trigeminal nucleus; MG, medial geniculate nuclei; MGP, medial globus pallidus; ml, medial lemniscus; mlf, medial longitudinal fasciculus; Mo5, motor trigeminal nucleus; MPB, medial parabrachial nucleus; MPO, medial preoptic nuclei; MS, medial septal nucleus; mt, mammillothalamic tract; NTS, * nucleus tractus solitarius; opt, optic tract; ox, optic chiasm; PAG, periaqueductal gray; PB, parabrachial nuclei; PBS, saline phosphate buffer; PBST, $0.1 \mathrm{M}$ saline phosphate buffer containing $0.3 \%$ Triton $\mathrm{X}-100$; PC, paracentral thalamic nucleus; PCom, nucleus of the posterior commisure; PCRt, parvicellular reticular nucleus; $\mathrm{Pe}$, periventricular hypothalamic nucleus; PF, parafascicular thalamic nucleus; PGi, paragigantocellular nucleus; $\mathrm{PH}$, posterior hypothalamic area; PHA-L, Phaseolus vulgaris-leucoagglutinin; $\mathrm{Pn}$, pontine nuclei; $\mathrm{PnC} / \mathrm{PnO} / \mathrm{PnV}$, pontine reticular nucleus caudal/oral/ventral part; $\mathrm{Po} / \mathrm{PoT}$, posterior thalamic nucleus/triangular part; $\mathrm{PO}$, preoptic nuclei; PPTg, pedunculopontine tegmental nucleus; $\mathrm{Pr}$, prepositus nucleus; PR, prerubral field; Pr5VL, principal sensory trigeminal nucleus, ventrolateral part; PV, paraventricular thalamic nuclei; PVN, * paraventricular hypothalamic nuclei; py, pyramidal tract/decussation; $R$, red nucleus; Re, reuniens thalamic nucleus; Rh, rhomboid thalamic nucleus; RMg, raphe magnus nucleus; $\mathrm{RPa} / \mathrm{ROb}$, raphe pallidus/raphe obscurus; $\mathrm{Rt}$, reticular thalamic nucleus; RVM, rostral ventromedial medulla; SC, superior colliculus; scp, superior cerebellar peduncle; SI, substantia innominata; SN, substantia nigra; SNC, substantia nigra compact part; SNR, substantia nigra reticular part; Sp5, spinal trigeminal nucleus; Sp5C, Sp5 caudal part; Sp5I, spinal trigeminal nucleus interpolar part; SPF, subparafascicular thalamic nucleus; VA, ventral anterior thalamic nucleus; VDB, nucleus of the vertical limb of the diagonal band; Ve, vestibular nuclei; VL, ventrolateral thalamic nucleus; VLH/VMH, ventrolateral/ventromedial hypothalamic nuclei; VLMlat, * lateral portion of the caudal ventrolateral medulla; VLPAG, ventrolateral periaqueductal gray; VM, ventromedial thalamic nucleus; VP, ventral pallidum; VPL/VPM, ventral posterolateral/ posteromedial thalamic nucleus; VRt, * ventral reticular nucleus; VTA/R, ventral tegmental area/rostral part; ZID/ZIV, zona incerta dorsal/ventral part; 3, oculomotor nucleus; 7 , facial nucleus; 10 , dorsal motor nucleus of vagus; 12 , hypoglossal nucleus; $3 \mathrm{~V}$, 3rd ventricle; $4 \mathrm{~V}$, 4th ventricle. 
nociceptive transmission can exert both an antinociceptive (inhibitory) and a pronociceptive (facilitating) action upon nociceptive spinal dorsal horn neurons (reviewed by Pertovaara, 2000; Lima and Almeida, 2002; Millan, 2002; Porreca et al., 2002; Gebhart, 2004). In this perspective, the final volume and the characteristics of peripheral nociceptive information reaching thalamic and cortical structures are dependent on the balance between these two opposing influences. Supraspinal brainstem areas like the periaqueductal gray matter (PAG; Bodnar, 2000), rostroventromedial medulla (RVM; Mason, 2001), locus coeruleus (LC; Jones, 1991), lateral portion of the caudal ventrolateral medulla (VLMlat; Tavares and Lima, 2002), dorsal reticular nucleus (DRt; Bouhassira et al., 1992) and nucleus tractus solitarius (NTS; Randich et al., 1988) are well established as being involved in antinociception. However, in recent years, the RVM (Porreca et al., 2002), the NTS (Wiertelak et al., 1997) and the DRt (Almeida et al., 1996; Almeida et al., 1999; Dugast et al., 2003) were shown to exert an additional profound nociceptive facilitating effect upon acute, inflammatory and/or chronic pain.

The DRt has been described in the caudalmost portion of the medullary dorsolateral reticular formation in rats (Valverde, 1962; Newman, 1985; Lima, 1990), monkeys (Villanueva et al., 1990) and humans (Koutcherov et al., 2004). To the best of our knowledge, anatomical, physiological and behavioral studies have solely explored the significant role of the DRt in pain processing and modulation (for reviews see Villanueva et al., 1996; Lima and Almeida, 2002; Monconduit et al., 2002). Anatomical studies have shown reciprocal connections between the DRt and the spinal dorsal horn laminae implicated in nociception (Almeida et al., 1993; Tavares and Lima, 1994; Almeida et al., 1995, 2000; Villanueva et al., 1995; Raboisson et al., 1996; Almeida and Lima, 1997). Electrophysiological studies revealed the presence in the DRt of a population of neurons activated exclusively or mainly by noxious stimuli applied to any part of the body (Villanueva et al., 1988, 1989). Functional studies on the activation of brain areas, measured by the consumption of 2-D-glucose, showed a significant activation of the DRt in inflammatory and chronic pain models (Neto et al., 1999; Porro, 2003). Finally, behavioral studies have implicated the DRt in pain exacerbation through a descending facilitating control of spinal nociceptive transmission in acute and inflammatory pain (Almeida et al., 1996, 1999; Dugast et al., 2003) and the depression of background body sensory activity (diffuse noxious inhibitory control, DNIC; Bouhassira et al., 1992).

The capacity to perform a fine modulatory action upon spinal nociceptive transmission requires a complex network of neuronal connections between brain areas implicated in ascending pain processing and descending pain modulation. Several studies have identified the brain connections of most pain control areas referred above, namely the PAG (Cameron et al., 1995a,b), RVM (Bobillier et al., 1976; Hermann et al., 1997), LC (Jones and Yang, 1985; Luppi et al., 1995), NTS (Menetrey and Basbaum, 1987; Arends et al., 1988; Joseph and Micheal, 1988) and VLMlat (Cobos et al., 2003; Babic et al., 2004). In the case of the DRt, although reciprocal connections with the spinal cord have been studied in detail (see above), only brain afferents to the nucleus have been thoroughly described (Almeida et al., 2002). Concerning efferent connectivity, some brain projections from the DRt have already been described (Bernard et al., 1990) as well as the DRt thalamic projections (Villanueva et al., 1998; Monconduit et al., 2002), both studies based on the anterograde tracer Phaseolus vulgaris-leucoagglutinin (PHA-L). However, recent preliminary studies using the anterograde tracer biotinylated dextran (BDA) have clearly shown a DRt brain projecting spectrum much broader then previously thought (LeiteAlmeida and Almeida, 2004).

In order to clarify the brain networks that can be used by the DRt to modulate the endogenous pain modulatory circuitry, a detailed analysis of the supraspinal areas receiving axonal projections from DRt was carried out by performing systematic injection of: (i) BDA into different DRt subareas; (ii) BDA in brain areas bordering the DRt; (iii) retrograde tracer cholera toxin subunit $B(C T b)$ into some of the DRt targets demonstrated anterogradely with BDA. Part of the data obtained was previously published in abstract form (Leite-Almeida and Almeida, 2004).

\section{EXPERIMENTAL PROCEDURES}

\section{Ethical guidelines}

Surgical procedures were performed under pentobarbital anesthesia $(50 \mathrm{mg} / \mathrm{kg}$, i.p.) on 31 Wistar male rats (Charles River Laboratories, Barcelona, Spain) weighing 280-320 g. Animals were placed in a stereotaxic device (Stoelting, Wood Dale, IL, USA) and a craniotomy was performed. Coordinates for brain injections followed the stereotaxic parameters of (Paxinos and Watson, 1998). The experiments were in accordance with the regulation of local authorities for handling laboratory animals and the European Community Council Directive 86/609/EEC. The number of animals used and their suffering were minimized.

\section{Anterograde tracing experiments}

Fifteen rats received iontophoretic injections (positive direct current of 2.5-3.0 $\mu \mathrm{A} ; 5 \mathrm{~s}$ on/5 s off, lasting for $10-30 \mathrm{~min}$ ) of $10 \% \mathrm{BDA}$ $(10,000 \mathrm{MW}$; Vector Laboratories, Burlingame, USA) in the left DRt through glass micropipettes with 15-20 $\mu \mathrm{m}$ diameter tips. After completion of the injection period the micropipettes were left in situ for 10-15 min before being slowly retracted to avoid tracer reflux along the pipette tract. Two to three weeks later, animals were reanesthetized with $35 \%$ chloral hydrate $(1 \mathrm{~mL} / \mathrm{kg}$ body weight) and perfused through the ascending aorta, first with $100 \mathrm{~mL}$ of saline phosphate buffer (PBS) $0,1 \mathrm{M}, \mathrm{pH} 7.2$ and then with $1000 \mathrm{~mL}$ of $4 \%$ paraformaldehyde in PBS. The entire brain was removed, immersed in the same fixative for $4 \mathrm{~h}$ and then in $8 \%$ sucrose in PBS at $4{ }^{\circ} \mathrm{C}$ for $1-2$ days. Coronal sections of the entire brain were serially cut on a vibratome at $50 \mu \mathrm{m}$ and incubated with $3.3 \% \mathrm{H}_{2} \mathrm{O}_{2}$ in order to inhibit endogenous peroxidase. Two in every three successive brain sections were immunoreacted with avidin-biotin complex (ABC, 1:200; Vector Laboratories) for $1 \mathrm{~h}$ and then BDA was revealed with 0.0125\% diaminobenzidine tetrahydrochloride (DAB; Sigma Immunochemicals, St. Louis, USA) and $0.02 \% \mathrm{H}_{2} \mathrm{O}_{2}$ in Tris-HCl buffer $0.05 \mathrm{M}, \mathrm{pH}$ 7.6. Half of these sections were counterstained using the formol-Thionin technique (Donovick, 1974) and the remaining were left without any counterstaining. Sections with and without counterstaining were then serially placed in SuperFrost Plus slides (MenzelGläser, Braunschweig, Germany), dehydrated and mounted in Entellan (Merck, Darmstadt, Germany). 


\section{Control experiments}

BDA administration to DRt adjacent nuclei. In order to determine the differential efferent pattern of medullary nuclei bordering the DRt, six animals received iontophoretic injections of BDA in the medullary ventral reticular nucleus (VRt; two rats), cuneate nucleus ( $\mathrm{Cu}$; two rats) and caudal spinal trigeminal nucleus (Sp5C; two rats). Injections were performed following the stereotaxic parameters of Paxinos and Watson (1998) and the same experimental procedures described above.

Retrograde-tracing studies. Ten Wistar male rats were injected with the retrograde tracer CTb (List Biological Products, Campbell, CA, USA) in areas receiving BDA-labeled terminal projections from the DRt as shown by the above anterograde injections in the nucleus. The areas selected were the medial (MPB, two rats) and lateral (LPB, two rat) parabrachial nuclei (PB), the ventrolateral periaqueductal gray matter (VLPAG, four rats) and the lateral globus pallidus (LGP, two rats). Animals were iontophoretically injected with $1 \%$ CTb using the same procedures described above for BDA experiments. 1 week after the injection they were reanesthetized and perfused as above. After the inhibition of endogenous peroxidase, serial brain sections were left overnight at room temperature in a goat antibody against CTb (List Biological Products) at $1: 40,000$ in 0.1 M PBS containing $0.3 \%$ Triton X-100 (PBST). After several washes in PBST sections were incubated for $1 \mathrm{~h}$ in PBST containing a biotinylated anti-goat antibody raised in horse (1:200; Vector Laboratories). Sections were washed again in PBST and then incubated in PBST containing $A B C(1: 200)$. This and subsequent steps were similar to those described above for BDA experiments.

\section{Image analysis and illustrations}

All the photographic material presented in this study was obtained using a digital camera (AxioCam HRc) connected to a microscope (Axioskop 2 Plus), both from Carl Zeiss (Göttingen, Germany). Images were captured in a computer using AxioVision 3.1.2.1 software and the brightness/contrast of each image was improved using Adobe Photoshop 7.0.1. software.

For illustrative proposes, the brain areas receiving efferent projections from the DRt were drawn using a sequence of selected formol-Thionin-stained coronal sections of one illustrative animal (rat 71; Figs. 1D, 2B) injected with BDA (Fig. 3). A motorized microscope (Axioplan2, Carl Zeiss) connected to a digital camera (Sony 3CCd DSP, Japan) was used to capture the image of the selected brain sections. For each section, the limits of the brain nuclei and the labeled fibers were drawn under a $1.25 \times$ or $40 \times$ objective lens, respectively, using Stereo Investigator 4.34 software (MicroBrightField, Inc, Willinston, VT, USA). It should be noted that these drawings do not distinguish whether fibers are terminal or main trunk profiles. This difference is discriminated in Table 1, which indicates the classification of the degree of axonal terminal arborization present in each brain area. This ranking system takes into account not only the amount of labeled terminals but also its consistent appearance throughout successive serial sections where a given nucleus is present. Therefore, the densities of fibers/ terminals in some areas represented in single section drawings might not reflect the scores attributed to the same area in Table 1.

The nomenclature/abbreviations used to designate brain nuclei and fiber tracts, except for a few exceptions assigned with $\left(^{*}\right)$ in the Abbreviations, are in accordance with those used by Paxinos and Watson $(1998,2005)$ or result from a simplification of it. For example, we have chosen $V e$ as a general designation for vestibular nuclei since we found no need to specify any particular nucleus of this area.
RESULTS

\section{Injection sites}

BDA injection sites appeared as a dark compact area surrounded by a halo constituted by a great amount of labeled fiber terminals. Some perikarya resulting from retrograde transport of the tracer were also present nearby the injection core. Only those injections whose central dark core and surrounding halo were located inside the DRt (Fig. 1 and Fig. 2A, B), VRt (Fig. 2C), Cu (Fig. 2E) and Sp5C (Fig. 2G) boundaries were considered valid for the present study. Selection of CTb injections (Fig. 2D, F, H) followed the same principle.

\section{Anterograde tracing experiments}

$B D A$ administration to the DRt. After BDA injections restricted to the $\mathrm{DRt}$, anterogradely labeled fibers and terminal boutons are present in the medulla oblongata (Fig. 3A-D), cerebellum (Fig. 3D), pons (Fig. 3E, F), mesencephalon (Fig. 3F, G), diencephalon (Fig. 3G-J) and in some restricted areas of the telencephalon (Fig. 3I-M). DRt neurons project bilaterally with ipsilateral prevalence to medullary, cerebellar, pontine and mesencephalic nuclei and with a contralateral predominance to diencephalic and telencephalic areas (Table 1; Fig. 3 ). Labeled fibers coursing rostrally are clustered in a restricted number of tracts: in the ipsilateral side they were confined to the medial longitudinal fasciculus (mlf; Fig. 3E), whereas in the contralateral side they course in the pyramidal tract (py; Fig. 3A, B) and medial lemniscus (ml; Fig. 3F, G). A detailed analysis of the brain areas receiving projections from the DRt is listed in Table 1.

Medulla oblongata. The largest amount of DRt fibers terminated in the medulla oblongata (Table 1; Fig. 3A-D). In its caudal aspect, a large number of labeled boutons were scattered through all the following ipsilateral reticular areas: DRt, lateral reticular nucleus (LRt), VRt and VLMlat. The equivalent contralateral areas were also supplied with a considerable amount of labeled terminals and fibers that decussate close to the central canal (Fig. 4A, B). Apart from the reticular formation, the motoneuronal pools concerned with orofacial motility, i.e. hypoglossal (12; Fig. 4A), trigeminal (Mo5, see Pons) and facial (7) nuclei, were the preferential targets of DRt efferents. At this level, only two restricted areas of the contralateral medial inferior olive (IOM) received similar amount of fibers (Fig. 3C), constituting the sole exception to the ipsilateral prevalence of DRt projections to the medulla oblongata. Medullary sensory areas receiving a moderate fiber projection from the DRt were the $\mathrm{Cu}$, external cuneate $(\mathrm{ECu})$, gracile $(\mathrm{Gr})$, NTS (Fig. 3A-C; 4A) and trigeminal nuclei. In the spinal trigeminal nuclei (Sp5; Fig. $3 \mathrm{~A}-\mathrm{C}$ ) fibers were mainly concentrated in the superficial laminae but a small number of terminals can also be detected in deeper trigeminal areas. DRt fibers spanned rostrally in a column-like fashion through all the rostrocaudal extension of the ipsilateral parvicellular reticular nucleus (PCRt), until the level of the $\mathrm{PB}$. The gigantocellular reticular nucleus $(\mathrm{Gi})$ and the RVM 


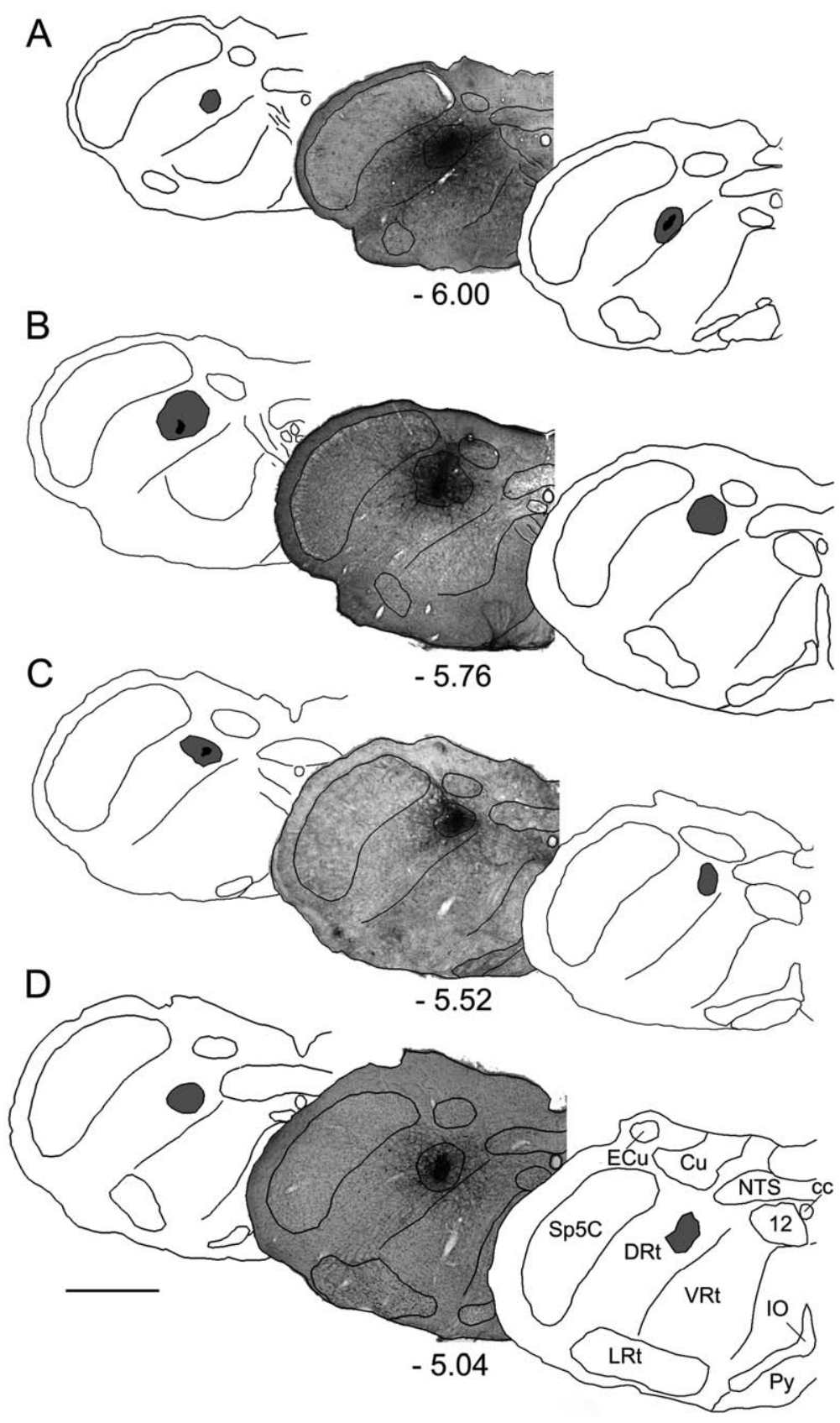

Fig. 1. Camera lucida-like drawings of representative BDA injections along four successive caudo-rostral levels of the DRt. Figures A-D correspond, respectively, to rats $84,79,36$ and 71 . Three drawings referring to the rostrocaudal extension of BDA administration are depicted for each injection. In the center, drawings and the corresponding photomicrograph are superimposed. Black areas represent the core of the injection whereas gray areas indicate the secondary labeling halo that surrounds the injection core. This code is not used when drawings and photomicrographs are superimposed. The number under the central image refers to the approximate rostrocaudal level based on the distance to the interaural line. Scale bar $=1 \mathrm{~mm}$.

[Gi alpha (GiA), lateral paragigantocellular (LPGi) and raphe magnus (RMg) nuclei] received moderate and consistent projections from the DRt (Figs. 3C-D; 5A).

Cerebellum. Bilateral DRt projections to the deep cerebellar nuclei of the cerebellum (Table 1; Fig. 3D) were present especially in the lateral (Lat) and interposed (Int) cerebellar nuclei. No labeled fibers or boutons were detected in the cerebellar cortex.
Pons. In the pons (Table 1; Fig. 3E, F), the Mo5 and the mesencephalic trigeminal (Me5) groups were targeted by a moderate and large amount of DRt fibers, respectively. Medially to Me5, the LC and/or the pericerulear area were also recipients of a small (contralateral) to medium (ipsilateral) amount of DRt projecting fibers. This same pattern was repeated for projections to the pontine lateral tegmental noradrenergic cell groups $A 5$ and A7. The PB 

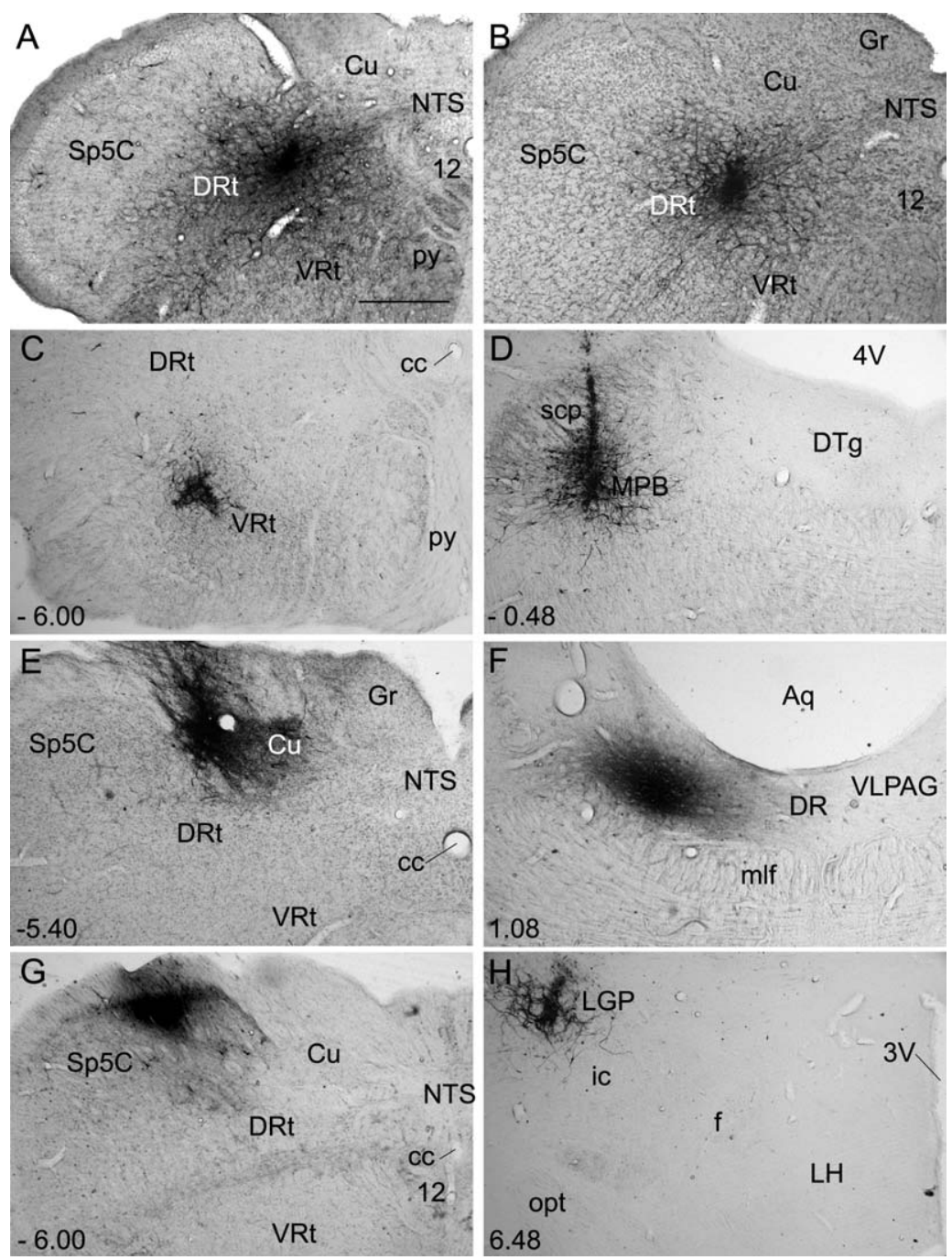

Fig. 2. Photomicrographs of selected areas injected with BDA ( $A, B, C, E$ and $G$ ) or $C T b(D, F$ and $H)$. Photomicrographs $A$ and $B$ correspond, respectively, to a caudal and a rostral injection of BDA in the DRt. The relationship to nuclei bordering the DRt is easily depicted in these sections stained by the formol-Thionin method. Note that $A$ and $B$ correspond to higher magnifications of, respectively, Fig. 1A and 1D. Photomicrographs $C$, $E, G$ and $D, F, H$ correspond, respectively, to anterograde and retrograde controls. Note that BDA injections are confined to the VRt (C), Cu (E) and Sp5C (G), whereas CTb injections are restricted to the MPB (D), VLPAG (F) and MGP $(H)$. The numbers at the lower left of panels C-F indicate the approximate rostrocaudal level based on the distance to the interaural line. Scale bar $=500 \mu \mathrm{m}$.

surrounding the superior cerebellar peduncle (scp) was richly supplied bilaterally by DRt efferents, especially the MPB and the ventrolaterally located Kölliker-Fuse nucleus $(\mathrm{KF})$. To a lesser extent, DRt terminals were also present in several pontine areas, including the pontine nucleus $(\mathrm{Pn})$ and different parts of the pontine reticular nucleus.

Mesencephalon. In the mesencephalon (Table 1; Fig. 3F, G), labeled fibers originated in the DRt were consistently present along the PAG, in gradients ranging from moderate in caudal areas to small in rostral areas, being the VLPAG the main target of DRt projections (Fig. $5 B)$. Other nuclei in PAG vicinity, like the dorsal raphe (DR), interstitial nucleus of the medial longitudinal fasciculus (IMLF), posterior commissure (PCom), oculomotor (3) and Darkschewitsch (DK) nuclei, received a smaller amount of fibers. A moderate number of labeled terminals were also present in the substantia nigra (SN; Fig. 6A, B), being concentrated almost exclusively in its compact (SNC) part. Additionally, small to moderate densities of labeled terminals were bilaterally present in the deep mesencephalic nucleus (DpMe), some rubral areas, ventral tegmental area (VTA) and both colliculi.

Diencephalon. DRt projections to the diencephalon were mainly contralateral and targeted a restricted number of thalamic, subthalamic and hypothalamic nuclei (Table 1; Fig. $3 G-J)$. The most prominent DRt projections to the thalamus terminated in the posterior (Po), parafascicular (PF), ventromedial (VM), and paracentral (PC) thalamic nuclei. Smaller amounts of labeled terminals were also present in the centromedial $(\mathrm{CM})$, centrolateral $(\mathrm{CL})$, reuniens $(\mathrm{Re})$, rhomboid $(\mathrm{Rh})$, ventrolateral (VL), ethmoid (Eth) and subparafascicular (SPF) nuclei. In the case of the hypothalamus, labeled fibers were especially concentrated in the lateral area (LH) and, in smaller amounts, in the posterior nucleus (PH). BDA filled 

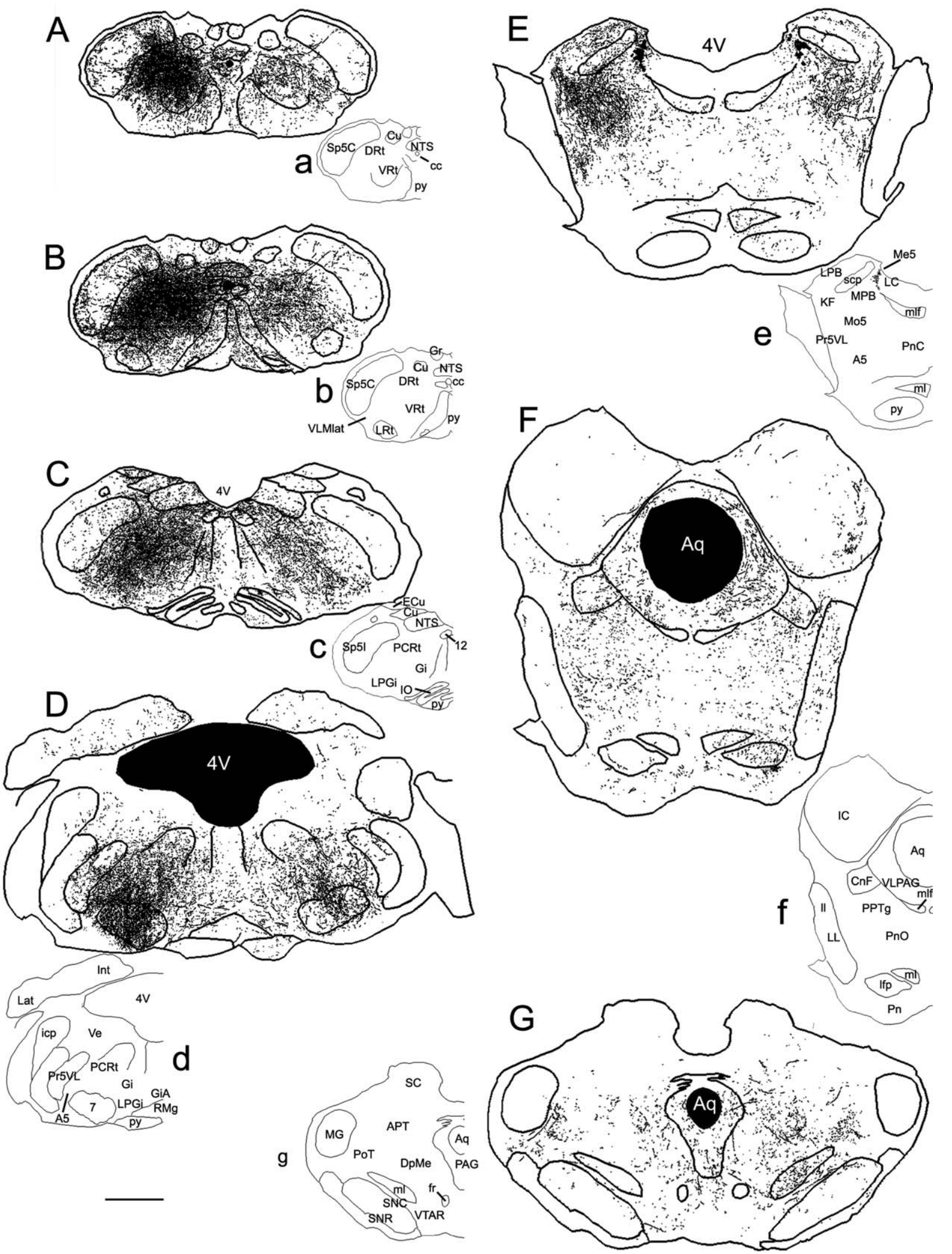

Fig. 3. Camera lucida-like drawings of selected sections representing the density of BDA-labeled fibers resulting from an unilateral injection of the tracer in the left DRt. All drawings refer to the same brain (rat 78) and are arranged from caudal (A) to rostral (M) coronal planes. Exceptionally, drawing $\mathrm{J} 2$ depicts a detail of the Amy from rat 71. Close to each drawing, and indicated with the equivalent small-case letter, it is depicted the corresponding hemisection showing the nuclei identification. Note the massive labeling along the extension of brainstem reticular formation (especially in the ipsilateral side) and in some areas of the diencephalon (mostly in the contralateral side). Conversely, in the forebrain the number of labeled terminals per brain section is low but it was found to be systematically present along the rostracaudal extension of some telencephalic areas as the MGP and VDB/HDB. Scale bar $=1 \mathrm{~mm}$. 


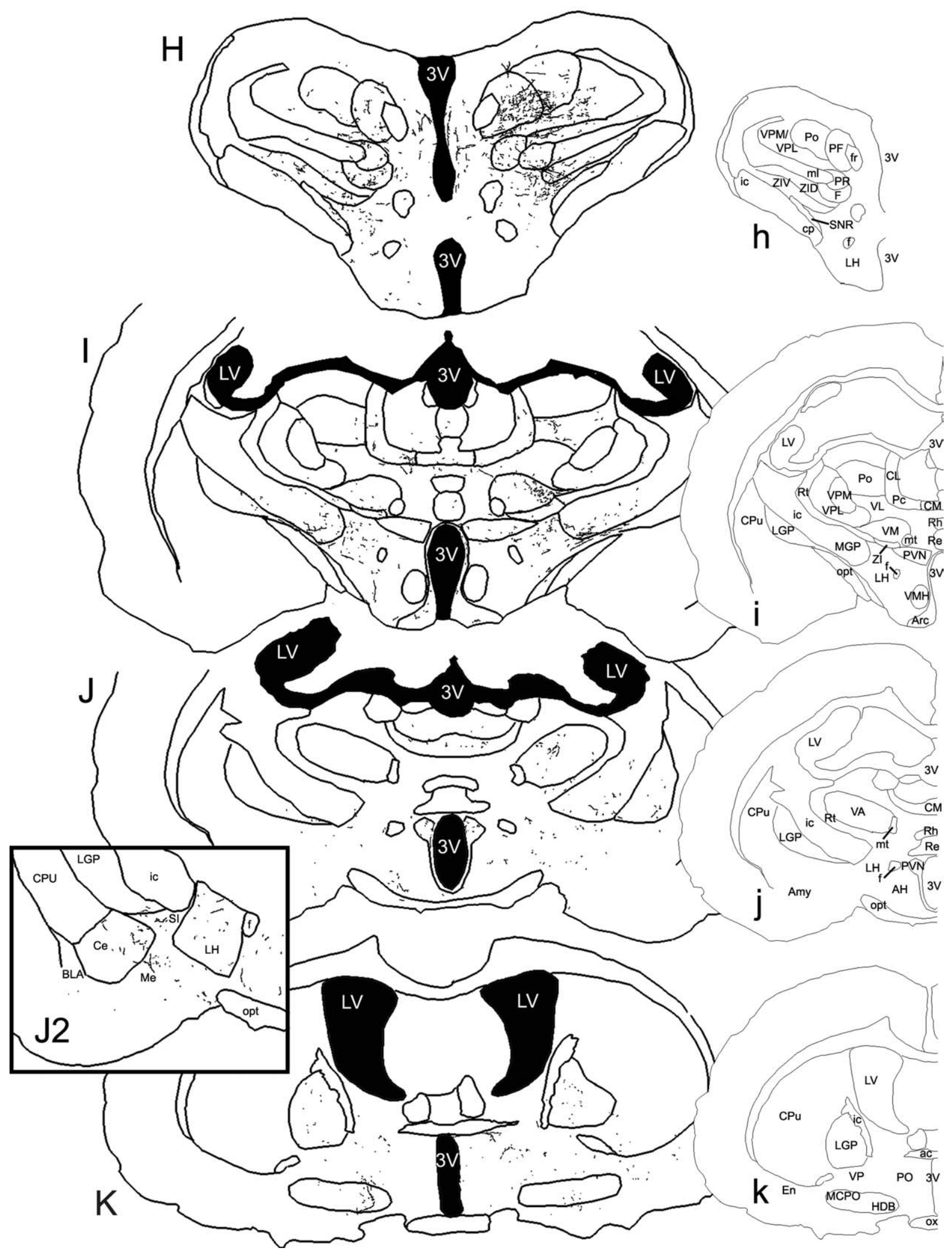

Fig. 3. (Continued).

terminals were also present in nuclei close to the 3rd ventricle, namely the arcuate (Arc), the periventricular $(\mathrm{Pe})$ and the paraventricular (PVN; Fig. 6C, D) nuclei, and in some preoptic nuclei. The dorsal (ZID) and ventral (ZIV) portions of the 


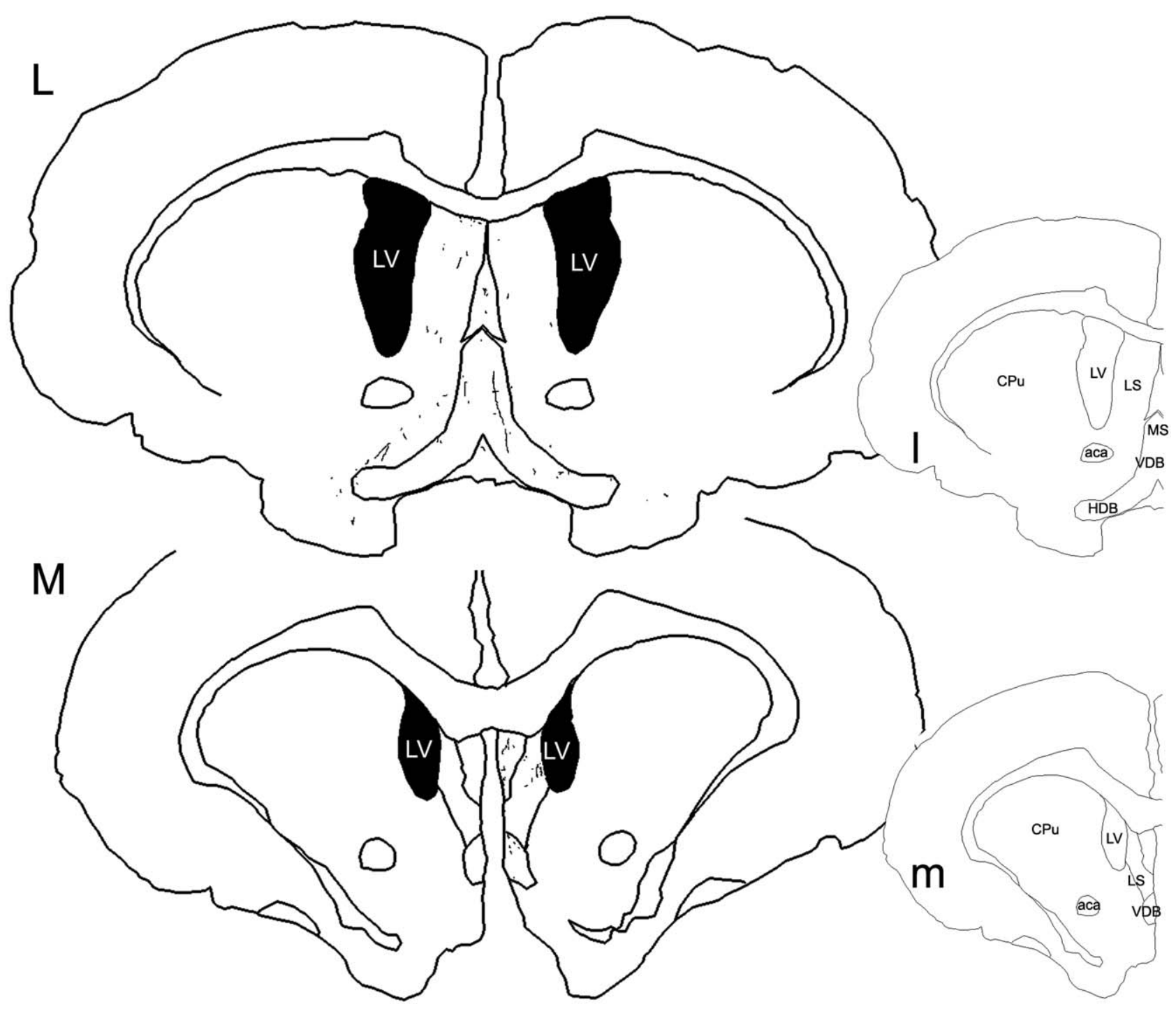

Fig. 3. (Continued).

subthalamic zona incerta were targeted by a moderate to large amount of fibers.

Telencephalon. BDA injections in the DRt resulted in labeled fibers in telencephalic areas (Table 1; Fig. 3J-M), especially in the MGP (see Fig. 6E, F) and, in smaller amounts, the LGP. Septal nuclei including the lateral (LSI) and medial (MS) areas, the indusium griseum (IG) and the nucleus of the vertical/horizontal limbs of the diagonal band of Broca (VDB/HDB; Fig. 6G, H) were also found to be targeted by DRt fibers. Only in two injections (rat 71, see Fig. 3J2) labeled terminals were present in amygdaloid nuclei (Amy), especially in the central $(\mathrm{Ce})$ and medial $(\mathrm{Me})$ ipsilateral Amy (Fig. 3J2). In these telencephalic areas, fiber distribution was predominantly contralateral and the number of labeled terminals per $50 \mu \mathrm{m}$ brain section was consistently present along the rostrocaudal axis of the recipient areas.

$B D A$ administration to nuclei bordering the DRt. The administration of BDA in medullary areas bordering the DRt, namely in the VRt (Fig. 2C), Cu (Fig. 2E) and Sp5C
(Fig. 2G), resulted in brain projecting patterns that were clearly different from those obtained after BDA injections in the DRt. See Table 1 for a detailed list of the areas receiving projections from these three nuclei.

VRt. BDA injections in the VRt (Fig. 2C; Table 1) resulted in a great number of labeled terminals in both ipsiand contralateral sides of the caudal medulla, including the NTS and reticular formation areas such us the intermediate reticular nucleus (IRt), LRt, VLMlat and, to a lesser extend, the DRt. In the rostral medulla, the RVM received also a considerable projection. At the pons level, VRt projections were mostly concentrated in the PB, KF and, at the mesencephalic level, the PAG was the major receptive area. The $\mathrm{LH}$ and $\mathrm{PH}$ hypothalamic nuclei and the VPL/ $\mathrm{VPH}, \mathrm{Po}$ and VM thalamic nuclei were the diencephalic areas to where the VRt projected more prominently. Lastly, the VDB/HDB is the only telencephalic area that receives a significant amount of projections from the VRt. Rare or no projections were found in the 12, ECu, Gi, PCRt, Sp5, 
Table 1. Density of DRt projections to the brain after BDA injections (rats 71 and 78 ) in the nucleus

\begin{tabular}{|c|c|c|c|c|c|c|c|c|c|c|}
\hline \multirow{3}{*}{$\begin{array}{l}\text { Location of labeled } \\
\text { terminals }\end{array}$} & \multicolumn{4}{|c|}{ DRt injections } & \multicolumn{6}{|l|}{ Controls } \\
\hline & \multicolumn{2}{|l|}{ Rat 71} & \multicolumn{2}{|l|}{ Rat 78} & \multicolumn{2}{|l|}{$\mathrm{Cu}$} & \multicolumn{2}{|l|}{ Sp5c } & \multicolumn{2}{|l|}{ VRt } \\
\hline & Ipsi & Cont & Ipsi & Cont & Ipsi & Cont & Ipsi & Cont & Ipsi & Cont \\
\hline \multicolumn{11}{|l|}{ Medulla oblongata } \\
\hline 7 & +++++ & ++++ & +++++ & ++++ & + & - & - & - & ++++ & + \\
\hline 10 & ++ & + & ++ & + & ++ & - & ++ & + & ++ & ++ \\
\hline 12 & +++++ & +++ & +++++ & +++ & ++ & - & ++ & & - & - \\
\hline $\mathrm{Cu}$ & ++ & + & +++ & + & Injection & + & ++ & - & + & - \\
\hline DRt & Injection & +++ & Injection & +++ & ++ & - & +++ & ++ & ++ & + \\
\hline DPGi & +++ & ++ & +++ & ++ & - & - & - & - & ++ & + \\
\hline $\mathrm{ECu}$ & +++ & - & +++ & - & +++++ & + & - & - & - & - \\
\hline $\mathrm{Gi}$ & +++ & ++ & ++++ & +++ & + & - & + & ++ & + & - \\
\hline $\mathrm{GiA}$ & ++ & ++ & - & - & + & +++ & - & - & +++ & ++ \\
\hline GiV & + & ++ & - & - & - & - & - & - & ++ & + \\
\hline $\mathrm{Gr}$ & ++ & + & ++ & + & +++ & ++ & ++ & + & ++ & - \\
\hline 10 & ++ & ++++ & + & +++++ & +++ & ++++ & - & - & + & + \\
\hline IRt & ++++ & +++ & ++++ & +++ & - & - & +++ & + & +++ & +++ \\
\hline LPGi & ++ & + & ++++ & +++ & ++ & + & + & - & ++ & +++ \\
\hline LRt & +++ & ++ & ++++ & +++ & ++ & - & + & - & +++ & ++++ \\
\hline NTS & +++ & ++ & +++ & ++ & ++ & + & ++++ & ++ & +++ & +++ \\
\hline PCRt & ++++ & +++ & ++++ & +++ & - & - & +++ & + & + & - \\
\hline $\mathrm{RMg}$ & +++ & +++ & +++ & ++ & + & + & - & - & ++ & ++ \\
\hline $\mathrm{ROb}$ & ++ & & ++ & & - & & - & & + & \\
\hline $\mathrm{RPa}$ & + & & ++ & & + & & - & & + & \\
\hline Sp5 & +++ & ++ & +++ & ++ & ++++ & ++ & Injection & + & + & - \\
\hline Ve & ++ & + & + & - & ++ & - & ++ & - & - & - \\
\hline VLMlat & +++ & +++ & +++ & ++ & +++ & + & ++++ & ++ & ++ & ++ \\
\hline VRt & +++ & +++ & ++++ & +++ & + & - & + & - & Injection & +++ \\
\hline \multicolumn{11}{|l|}{ Cerebellum } \\
\hline Cortex & - & - & - & - & ++++ & ++ & +++ & - & - & - \\
\hline Lat/Int & ++ & + & ++ & ++ & - & - & - & - & + & + \\
\hline \multicolumn{11}{|l|}{ Pons } \\
\hline A5 & ++++ & + & +++ & ++ & ++ & - & - & - & ++ & - \\
\hline $\mathrm{KF}$ & +++ & ++ & ++++ & +++ & + & - & +++ & +++ & ++++ & ++ \\
\hline LC & +++ & + & ++ & + & ++ & - & - & - & ++ & ++ \\
\hline LL & + & +++ & ++ & + & - & +++ & - & ++ & - & - \\
\hline Me5 & ++ & ++ & ++ & ++ & - & - & - & - & - & - \\
\hline Mo5 & ++++ & +++ & ++++ & +++ & - & - & - & - & ++ & + \\
\hline PB & ++++ & ++ & ++++ & +++ & + & - & ++++ & +++ & ++++ & ++ \\
\hline $\mathrm{Pn}$ & + & ++ & ++ & ++ & - & +++++ & - & - & - & - \\
\hline $\mathrm{PnC} / \mathrm{PnO}$ & ++ & + & +++ & ++ & - & +++ & + & + & ++ & + \\
\hline PnV & - & ++ & - & - & - & - & - & - & - & - \\
\hline \multicolumn{11}{|l|}{ Mesencephalon } \\
\hline 3 & ++ & ++ & + & ++ & - & - & - & - & + & + \\
\hline APT & - & ++ & - & ++ & + & ++ & - & - & ++ & - \\
\hline $\mathrm{CnF}$ & ++ & ++ & ++ & ++ & - & - & - & - & - & - \\
\hline Colliculi & ++ & +++ & +++ & ++ & - & - & - & +++ & ++ & \\
\hline DK & - & ++ & ++ & ++ & - & + & - & - & - & - \\
\hline DR & ++ & ++ & +++ & +++ & - & - & - & - & - & - \\
\hline DpMe & ++ & +++ & ++ & +++ & - & +++ & - & + & + & + \\
\hline $\mathrm{F}$ & ++ & ++ & +++ & ++ & - & +++ & - & - & - & - \\
\hline IMLF & ++ & ++ & ++ & ++ & - & ++ & - & - & - & - \\
\hline IP & ++ & - & + & + & - & - & - & - & - & - \\
\hline PAG & +++ & ++ & +++ & +++ & - & ++ & - & - & +++ & +++ \\
\hline $\mathrm{R}$ & +++ & +++ & ++ & ++ & - & + & - & - & ++ & ++ \\
\hline SNC & ++ & ++ & + & +++ & - & + & - & - & + & + \\
\hline SNR & + & - & - & + & - & - & - & - & - & - \\
\hline VTA & ++ & ++ & ++ & ++ & - & + & - & - & - & - \\
\hline
\end{tabular}


Table 1. Continued

\begin{tabular}{|c|c|c|c|c|c|c|c|c|c|c|}
\hline \multirow{3}{*}{$\begin{array}{l}\text { Location of labeled } \\
\text { terminals }\end{array}$} & \multicolumn{4}{|c|}{ DRt injections } & \multicolumn{6}{|c|}{ Controls } \\
\hline & \multicolumn{2}{|l|}{ Rat 71} & \multicolumn{2}{|l|}{ Rat 78} & \multicolumn{2}{|l|}{$\mathrm{Cu}$} & \multicolumn{2}{|c|}{ Sp5c } & \multicolumn{2}{|l|}{ VRt } \\
\hline & Ipsi & Cont & Ipsi & Cont & Ipsi & Cont & Ipsi & Cont & Ipsi & Cont \\
\hline \multicolumn{11}{|l|}{ Diencephalon } \\
\hline \multicolumn{11}{|l|}{ Hypothalamus } \\
\hline Arc & +++ & ++ & +++ & ++ & - & - & - & - & - & - \\
\hline $\mathrm{LH}$ & +++ & +++ & +++ & +++ & - & ++ & - & - & - & - \\
\hline LM & ++ & ++ & + & + & - & - & - & - & - & ++ \\
\hline L/MPO & ++ & ++ & + & + & - & - & - & - & - & - \\
\hline $\mathrm{Pe}$ & ++ & ++ & ++ & ++ & - & - & - & - & - & - \\
\hline $\mathrm{PH}$ & ++ & ++ & ++ & ++ & ++ & ++++ & - & - & ++ & ++ \\
\hline PVN & ++ & - & + & ++ & - & - & - & - & - & + \\
\hline VLH/VMH & ++ & + & - & + & - & - & - & - & - & - \\
\hline \multicolumn{11}{|l|}{ Thalamus } \\
\hline $\mathrm{CL}$ & - & - & + & ++ & - & - & - & - & - & + \\
\hline $\mathrm{CM}$ & ++ & ++ & ++ & +++ & - & - & - & - & + & + \\
\hline Eth & - & ++ & - & ++ & - & - & - & - & - & - \\
\hline $\mathrm{PC}$ & ++ & +++ & ++ & +++ & - & - & - & - & - & - \\
\hline PF & +++ & +++ & +++ & +++ & - & ++ & - & - & - & + \\
\hline Po & +++ & ++++ & +++ & ++++ & - & +++ & - & +++ & - & ++ \\
\hline PV & - & ++ & - & + & - & - & - & - & - & - \\
\hline $\operatorname{Re}$ & - & - & + & - & - & - & - & - & - & - \\
\hline $\mathrm{Rh}$ & - & + & - & + & - & - & - & - & - & - \\
\hline $\mathrm{Rt}$ & - & - & + & + & - & - & - & - & - & - \\
\hline SPF & ++ & +++ & + & ++ & - & ++++ & - & ++ & + & + \\
\hline VA & - & - & + & + & - & - & - & - & - & - \\
\hline $\mathrm{VL}$ & + & + & + & ++ & - & - & - & - & - & + \\
\hline VM & - & +++ & + & +++ & - & - & - & - & + & ++ \\
\hline VPM/VPL & + & ++ & + & ++ & - & + & - & +++ & - & +++ \\
\hline \multicolumn{11}{|l|}{ Subthalamus } \\
\hline ZI & ++ & +++ & ++ & +++ & - & +++ & - & - & + & ++ \\
\hline \multicolumn{11}{|l|}{ Telencephalon } \\
\hline Amy & +++ & - & - & - & - & - & - & - & - & - \\
\hline BST & +++ & ++ & - & - & - & - & - & - & - & - \\
\hline IG & + & - & - & ++ & - & - & - & - & - & - \\
\hline GP (L/MPG) & + & ++ & ++ & +++ & - & - & - & - & - & + \\
\hline LSI & + & - & - & + & - & - & - & - & - & - \\
\hline MS & + & + & - & + & - & - & - & - & - & - \\
\hline $\mathrm{SHi}$ & - & - & - & + & - & - & - & - & - & - \\
\hline SI & ++ & + & + & ++ & - & - & - & - & - & + \\
\hline VDB/HDB & ++ & + & ++ & ++ & - & - & - & - & - & +++ \\
\hline VP & - & - & - & + & - & - & - & - & - & - \\
\hline
\end{tabular}

Results for control injections of BDA in nuclei surrounding the DRt, namely in the $\mathrm{Cu}, \mathrm{Sp} 5 \mathrm{C}$ and VRt are also shown for comparison of projection patterns. Intensity of labeling: +++++ , very dense; ++++ , dense; +++ , numerous; ++ , few; + , rare; - , no labeling.

Me5, DR, VTA, Arc, LH, L/MPO, PC, Amy, BST, which were strongly or moderately targeted by DRt efferents.

$\mathrm{Cu}$. At the most caudal level of the brainstem, $\mathrm{Cu}$ projections (Fig. 2E; Table 1) were mainly concentrated in the Sp5C, Gr and VLMlat. Further rostrally, the ipsilateral $\mathrm{ECu}$ was the strongest recipient of labeled terminals from all $\mathrm{Cu}$ efferent targets. The $\mathrm{IO}$ also received large quantities of fibers both ipsi- and contralaterally. In the cerebellum, terminal fibers from the $\mathrm{Cu}$ were present throughout the rostrocaudal extension of the ipsilateral cortex. At the pons, massive projections were especially concentrated in the $\mathrm{PnC} / \mathrm{PnO}$ and contralateral $\mathrm{Pn}$. Less significant amounts of fibers were also present in the LC, PB, KF and A5. At the mesencephalon, the principal $\mathrm{Cu}$ projections tar- geted the nucleus of the fields of Forel (F), DpMe, PAG and anterior pretectal nucleus (APT), contralaterally. Cu diencephalic projections to the hypothalamus terminated in the $\mathrm{LH}$, ipsilaterally and $\mathrm{PH}$, bilaterally. In the thalamus, all $\mathrm{Cu}$ projections were contralateral and targeted the Po, PF and the SPF nuclei. Labeled fibers were also present in the contralateral subthalamic Zl. No projections were found in the telencephalon and rare or terminal boutons were detected in the VRt, 7, Gi, GiV, PCRt, deep cerebellar nuclei, KF, Me5, Mo5, $\mathrm{PB}, \mathrm{CnF}$, colliculi, DR, SN, CM, PC, PF, VM, ZI and PVN, which were found to be targeted consistently by the DRt.

Sp5C. Sp5C projections (Fig. 2G; Table 1) to the caudal brainstem were especially concentrated in the NTS and VLMlat. Smaller amounts of labeled fibers were also 

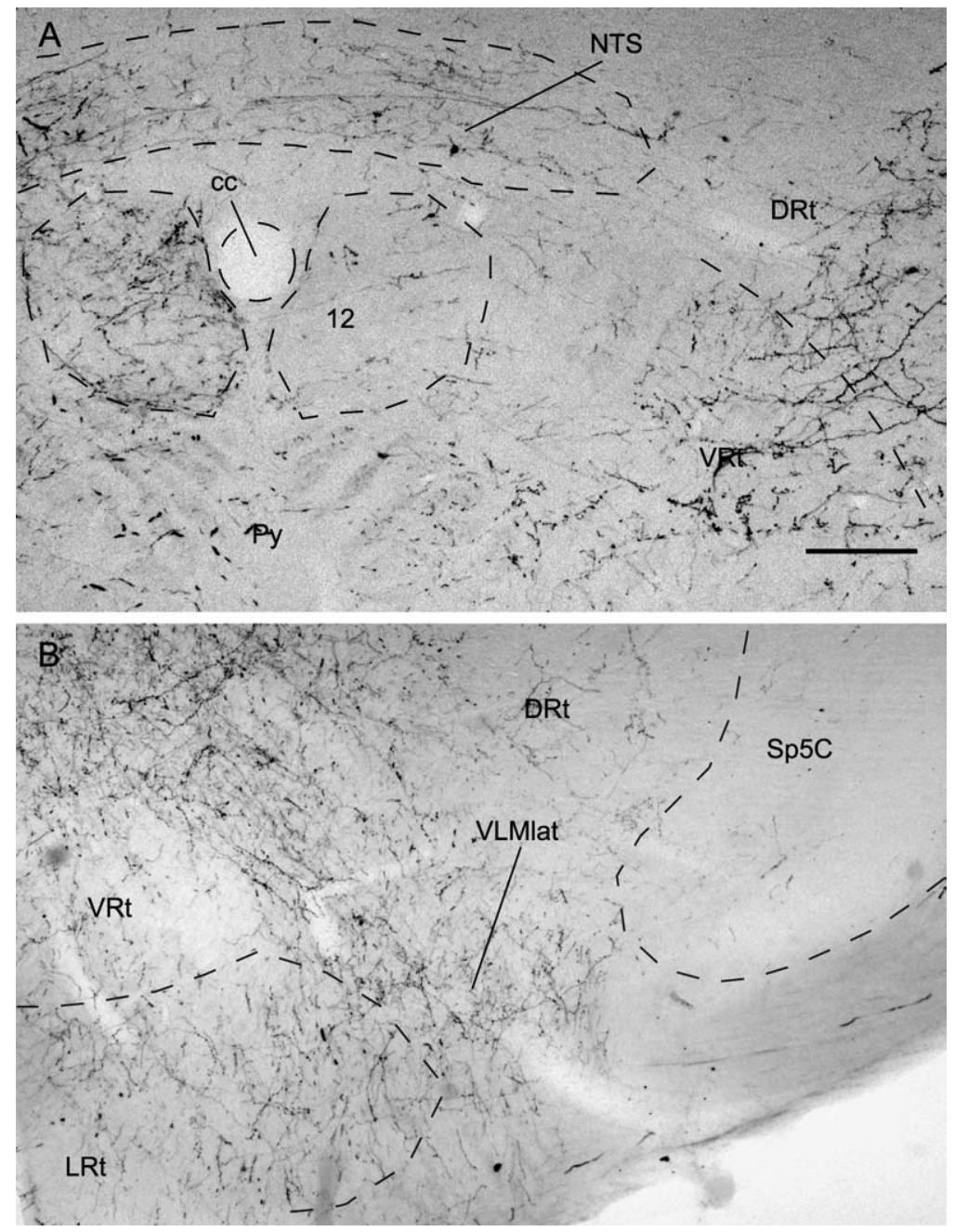

Fig. 4. Photomicrographs of BDA-labeled terminals in contralateral hemisections of the caudal brainstem. Some of these areas have been previously implicated in supraspinal pain modulation, namely the DRt (A, B), the VRt (A, B), the NTS (A) and the VLMlat (B). Note the great concentration of fibers in the ipsilateral 12 and the NTS bilaterally (A). Scale bar $=250 \mu \mathrm{m}$.

present in the DRt, IRt and $\mathrm{Cu}$. At the pons, both the ipsiand contralateral $\mathrm{PB}$ and $\mathrm{KF}$ received efferents from the Sp5C and, at the mesencephalon, only the ipsilateral colliculi presented fibers labeled from the Sp5C. At the diencephalon, projections from the Sp5C were restricted to the contralateral ventral posterolateral (VPL/VPM), Po and SPF thalamic nuclei. No labeled terminals were identified in other diencephalic areas as well as in the telencephalon. Rare or no projections were found in the 7, ECu, GiA, IO, LRt, RMg, ROb, VRt, deep cerebellar nuclei, A5, LC, Me5, Mo5, APT, CnF, DR, PAG, SN and VTA, areas that were consistently targeted by the DRt.

\section{Retrograde tracing experiments}

In order to verify the performance of the anterograde tracer BDA in the present work, three areas targeted by the DRt were selected for injections with the retrograde tracer CTb.

The PB was already known from a previous work (Bernard et al., 1990) to receive a moderate amount of fibers from the DRt and was therefore selected to test the effi- cacy of our retrograde experiments. Injections of $\mathrm{CTb}$ in the MPB (Fig. 2D) resulted in a relatively high amount of retrograde labeled cells distributed throughout all the rostrocaudal extension of the DRt (see Fig. 7E), especially in the ipsilateral hemisection.

The main brainstem area implicated in pain modulation, the PAG was also selected to receive CTb injections (Fig. 2F). These always resulted in a moderate to low number of retrogradely labeled neurons in the DRt (Fig. $7 A, B)$, thus confirming the anterograde result obtained with BDA. It was found that this number was highly dependent on the area injected, CTb injections in caudalmost PAG being those which resulted in a higher numbers of labeled neurons in the DRt (Fig. 7C, D).

Lastly, we have selected a telencephalic area targeted anterogradely by BDA injected in the DRt since no other study has ever shown a projection to this area of the brain. Injections of CTb in the MGP resulted in a small number of retrogradely labeled neurons in the DRt (Fig. 7F), confirming the results obtained with BDA. 

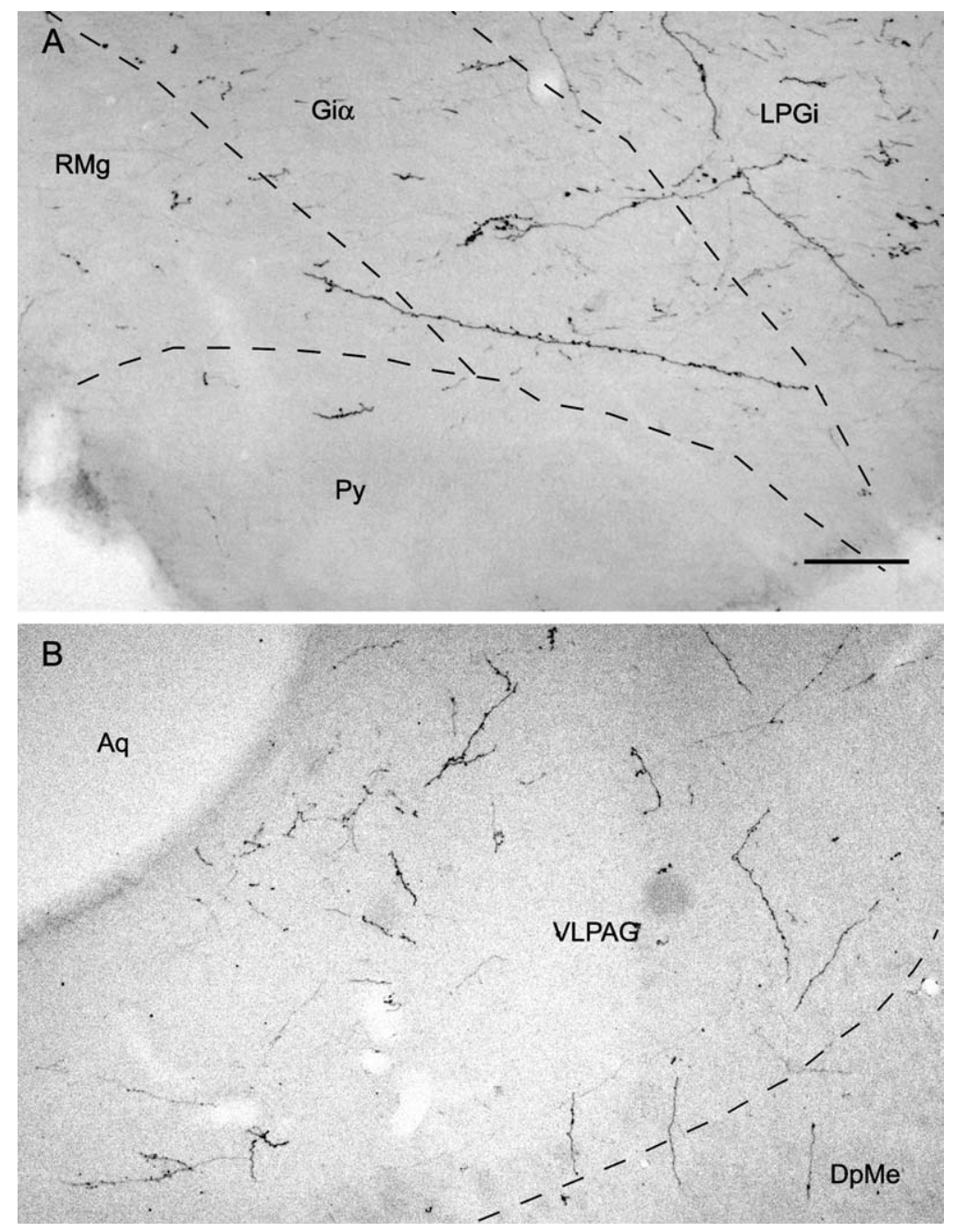

Fig. 5. Photomicrographs of BDA-labeled terminals in the contralateral RVM (A) and PAG (B), both areas being part of the major axis of the endogenous pain control system. Scale bar $=250 \mu \mathrm{m}$.

\section{DISCUSSION}

The present data demonstrate that the DRt projection pattern to the brain is broader than previously thought (Fig. 8; see for comparison Bernard et al., 1990). Importantly, some of the areas now described as receiving projections from the DRt are known for a long time to be implicated in pain modulation. These include all relay areas of the descending hypothalamus-PAG-RVM-dorsal horn circuitry, the noradrenergic cell groups LC, A5 and A7, the NTS and virtually all brainstem areas of the reticular formation involved in pain control. Additionally, projections to the medial thalamus and the limbic system are suggestive of a DRt role in the emotional processing of pain. Similarly, connections to several areas belonging to the extrapyramidal and orofacial motor system indicate an involvement in the motor reactions associated with pain. Taken together, these data reinforce the anatomical basis for the well-known role of the DRt as a medullary center devoted to pain processing and modulation (see reviews by Villanueva et al., 1996; Lima and Almeida, 2002).

\section{Technical considerations}

A certain number of principles were followed in order to validate our results, namely in what concerns the definition of DRt boundaries and the benefits and/or caveats of the tracers used in this study.

\section{Specificity of the DRt brain projection patterns}

Rostrocaudally, the DRt is located between the most rostral aspect of the lamina $V$ of the spinal dorsal horn, at the level of the pyramidal decussation and the most caudal aspect of the PCRt, at the level of the rostral end of the area postrema (AP; Valverde, 1962; Newman, 1985; Paxinos and Watson, 1998). On the coronal plane, the DRt boundaries are defined by the Cu dorsally, the Sp5C laterally, the NTS dorsomedially and the VRt/VLMlat ventromedially. These areas are functionally distinct as a reflex of their particular afferent and efferent connectivity, as shown by their sharply different efferent projection patterns when compared with that of the DRt (Table 1). The DRt has the highest projection to orofacial motor nuclei 12, 7 and Mo5, 

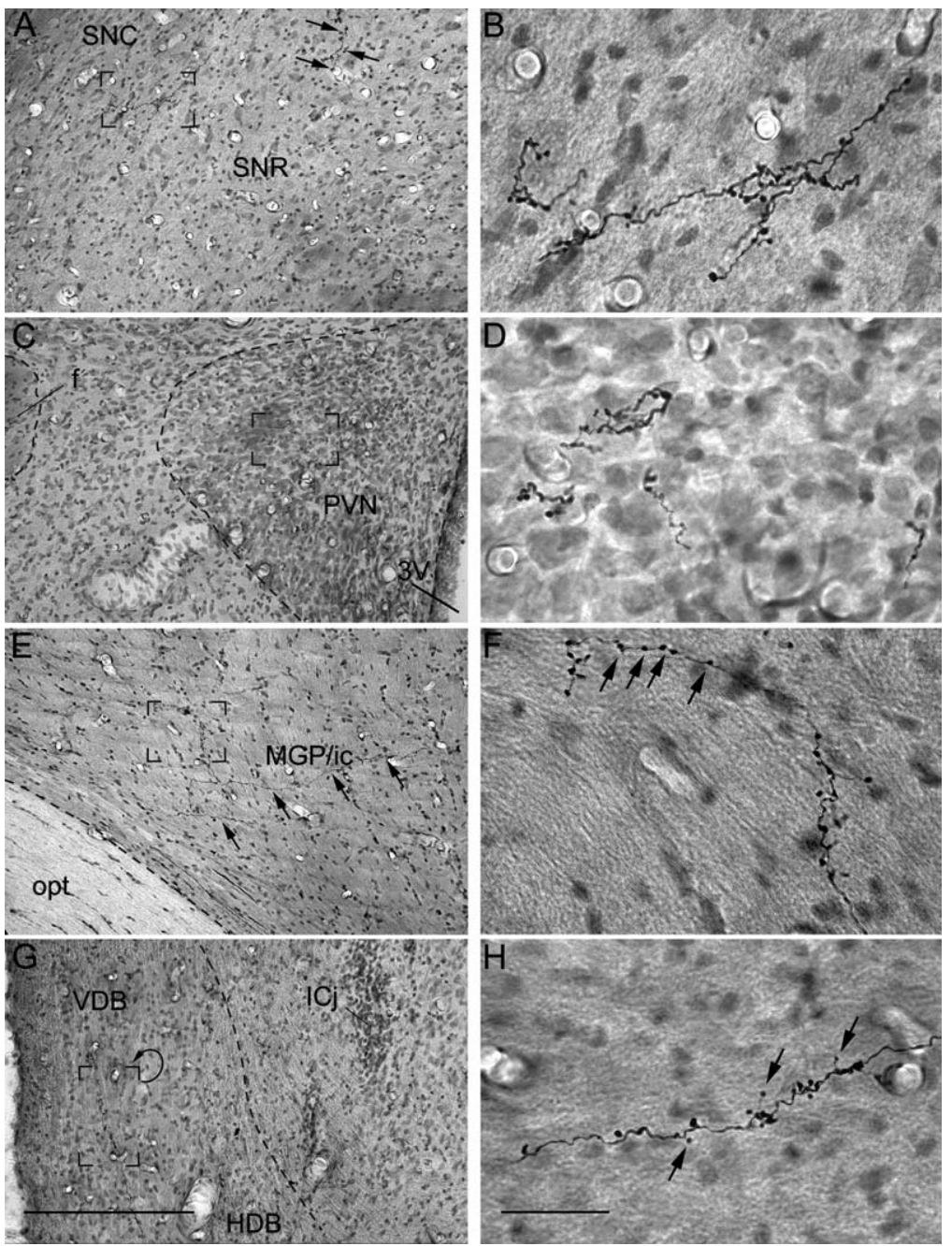

Fig. 6. Photomicrographs of BDA-labeled terminals in forebrain sections stained by the formol-Thionin method. The number of fibers was usually low in each $50 \mu \mathrm{m}$ brain section but they were consistently present along the rostrocaudal extension of the nuclei shown in the picture. The areas depicted are the contralateral SNC/SNR (A, B), the ipsilateral PVN (C, D), the ipsilateral MGP (E, F) and the contralateral VDB/HBD (G, H). The left column $(A, C, E, G)$ presents a low magnification of the brain area of interest. The areas assigned with a rectangle are shown in the correspondent high magnification images present in the right column (B, D, F, H). Additional labeled fibers outside the rectangle are indicated by arrows (A, E). In figure $\mathrm{G}$ the curving arrow indicates that the corresponding high magnification photomicrograph $(\mathrm{H})$ is rotated in a counterclockwise way. Arrows in the right column high magnification photomicrographs point out en passant varicosities $(F)$ and terminal boutons $(H)$. Note that in some higher magnifications different focal plans were used to create a single picture. These can be readily identified because of the slight different background gray intensities. Scale bars $=200 \mu \mathrm{m}$ left column; right column, $20 \mu \mathrm{m}$.

whereas the VRt does not project to the 12 and the $\mathrm{Cu}$ and Sp5C do not project to the Mo5. The presence of labeled terminals in the cerebellar cortex was detected after BDA injections in the $\mathrm{Cu}$ and, in smaller amounts, in the Sp5C but no injections confined to the limits of DRt or VRt were able to reproduce this result. At the pons level, projections from $\mathrm{Cu}$ to the $\mathrm{Pn}$ were more intense than those from the DRt, VRt and Sp5C but, on the contrary, the Cu was the only area that did not project to the PB. The efferent projection pattern to the PAG also differed among the four nuclei injected with BDA: contrary to injections confined to the DRt, VRt and Cu no labeled terminals were found in the PAG after BDA injections in the Sp5C and, in the case of $\mathrm{Cu}$, these projections were exclusively contralateral and especially located in rostral areas (as it was confirmed retrogradely; Fig. 7D). BDA injections in the Sp5C have not resulted in labeled terminals in the hypothalamus, contrary to what happened after injections in the other nuclei. Finally, only injections confined to DRt and VRt have resulted in labeled terminals at the telencephalic level. These data reinforce the clear-cut distinction between the results obtained by small well-delimited BDA injections applied to the DRt and neighbor brain areas.

\section{Specificity of the tract-tracing methodology}

Dextran amines like BDA are among the best commercially available tracers (Köbbert et al., 2000) and, at least in peripheral sensory pathways, BDA seems to be a more powerful anterograde tracer than PHA-L (Novikov, 2001). 

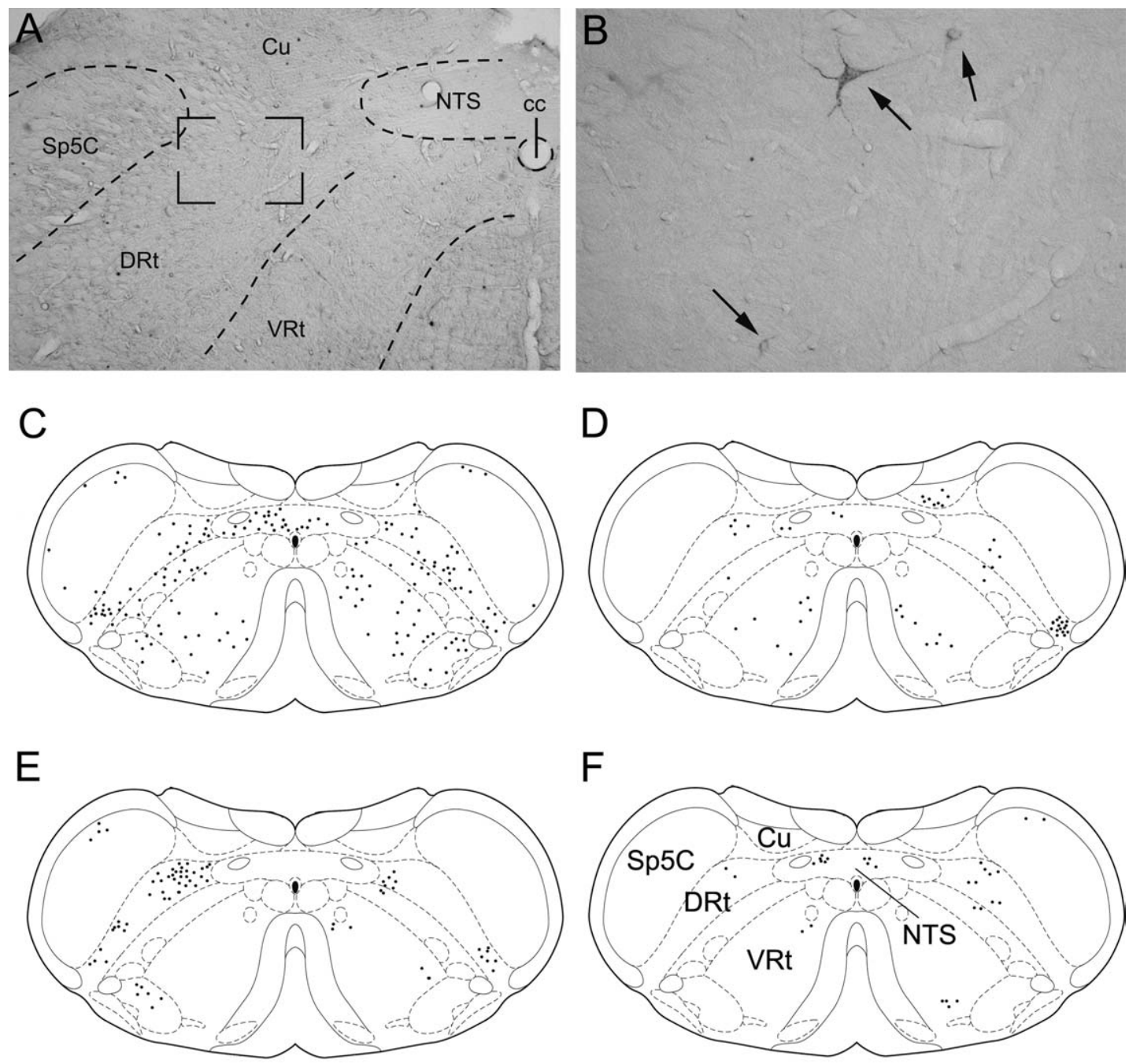

Fig. 7. Photomicrographs (A, B) showing retrogradely labeled neurons in the DRt after the VLPAG injection of CTb presented in Fig. 3E. The low magnification micrograph (A) shows the local anatomy of the caudal medulla and retrogradely labeled in the area. High magnification (B) micrograph shows the area inside the rectangle in A. (C-F) Schemes adapted from Paxinos and Watson (2005) representing the central zone of the rostrocaudal extension of the DRt; CTb retrogradely labeled neurons from 15 to 20 sections (50 $\mu \mathrm{m}$ thickness each one separated by $150 \mu \mathrm{m})$ are represented by a single dot after tracer injection in the caudal VLPAG (see injection Fig. 2F) (C) in the rostral VLPAG (D) in the MPB (see injection Fig. 2D) (E) and in the MGP (see injection Fig. 2H) (E). Scale bar=500 $\mu \mathrm{m}$ in A; B, $125 \mu \mathrm{m}$.

Although BDA can be incorporated into injured dendrites and/or fibers at the injection site, labeling of en passage fibers is reduced by small diameter micropipette tips during the iontophoretic administration. This was the case of the present study since virtually no labeled perikarya were found along the brain of injected rats. Curiously, we have found some discrepancies between our present data obtained with BDA injections in the DRt and the data obtained previously with PHA-L (see below; Bernard et al., 1990; Villanueva et al., 1998) which might be explained by different cellular incorporation/uptaking mechanisms of the tracers.

Our injections in the DRt resulted in a consistent efferent projection pattern to the brainstem, thalamus, caudal hypothalamus and nigral basal ganglia. Less consistently, we have found after some BDA injections DRt-labeled terminals in the rostral hypothalamus (preoptic area), basal nuclei MGP and LGP, substantia innominata (SI), some lateral and medial septal nuclei and, in two rats, in the Amy. In these cases some considerations should be made. Firstly, injections of anterograde tracers in several levels/ laminae of the spinal cord confirmed the existence of spinal projections to the above-mentioned areas (Cliffer et al., 1991; Wang et al., 1999; Gauriau and Bernard, 2004). This fact raises the question of whether our labeling in the forebrain is due to the caption of BDA by DRt neurons or by spinofugal en passage fibers coursing through the DRt. However, we reject this possibility: (i) injections of retrograde tracers in some of these forebrain areas confirmed the presence of small amounts of labeled cells in the DRt; (ii) fiber tracts ascending from the spinal cord are mostly concentrated in discreet areas of white matter located far from the injected DRt areas (Paxinos and Watson, 1998). Thus, it is possible that different neuronal populations co- 


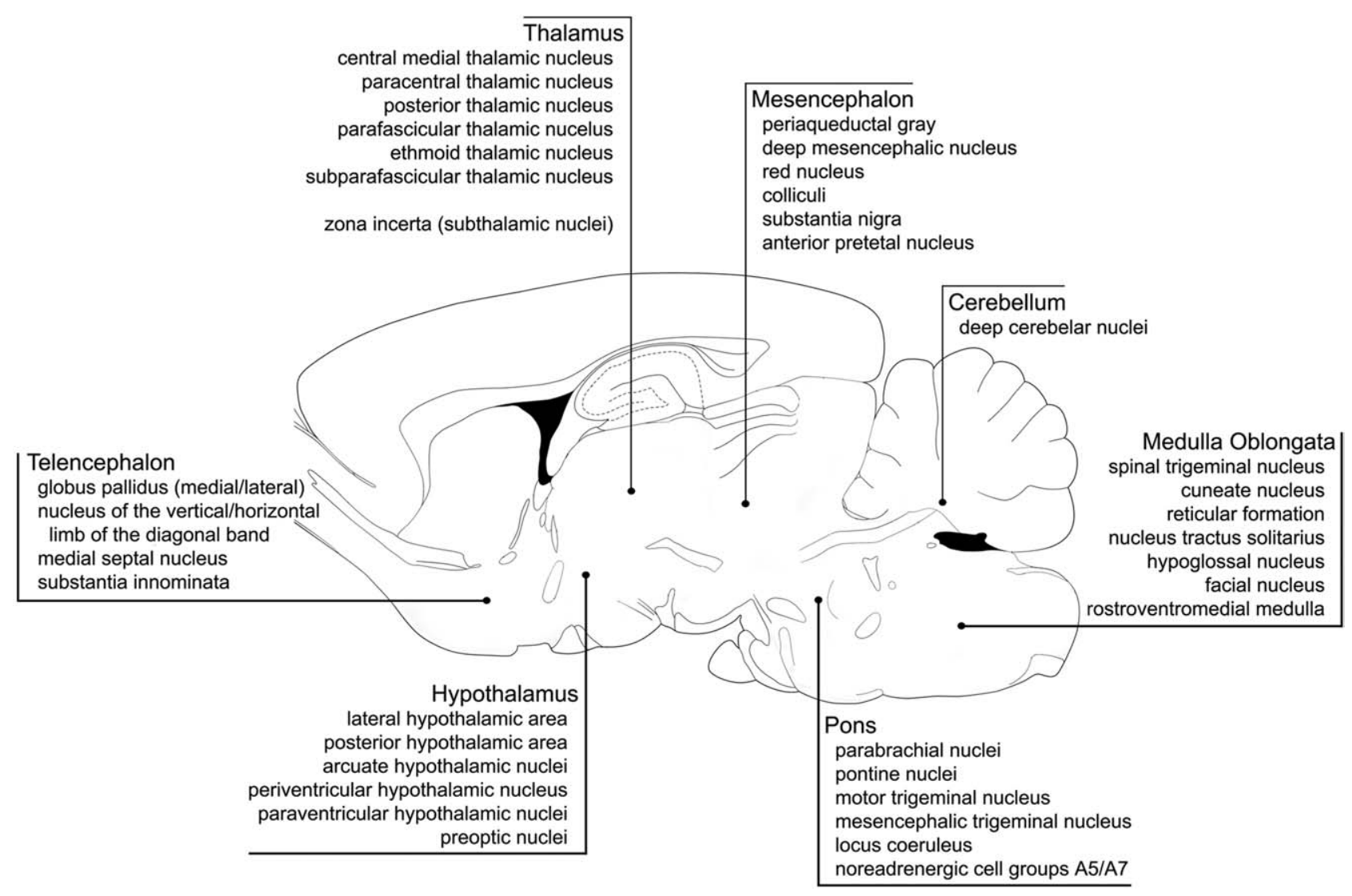

Fig. 8. Schematic diagram summarizing the main DRt projections to the brain. The saggital section is adapted from Paxinos and Watson (1998).

exist in the DRt. This hypothesis is supported by other retrograde (Li JL et al., 1997) and anterograde (Cliffer et al., 1991; Wang et al., 1999; Gauriau and Bernard, 2004) studies demonstrating the presence of forebrain-projecting neurons in the upper cervical levels and in the caudal medulla. Importantly, a similar hypothesis has been already ascribed to the DRt based on some intermittence occurring with a few afferent projections to this nucleus, which seemed to depend on local neuronal subpopulations (Almeida et al., 2002).

CTb has the advantage of not being picked up by fibers en passage (Lima and Coimbra, 1988; Tavares and Lima, 1994). Therefore, the presence of retrograde labeled cells in the DRt after a CTb injection in another brain area is a strong reinforcement that the latter is a DRt target. In fact, all CTb injections applied to previously BDA labeled areas resulted in perikarya labeling in the DRt.

\section{Anatomo-functional considerations}

Brainstem. The major targets of DRt projections are located in the brainstem. Fibers are distributed almost ubiquitously along the reticular formation, ranging from large amounts in areas such as the PCRt, NTS and LRt, to moderate or small in areas as the pontine reticular nuclei, cuneiform nucleus (CnF), DpMe, VTA (confirmed retrogradely by Herbert et al., 1997) and both colliculi. Except for the PCRt and Gi (Bernard et al., 1990), none of the other nuclei had been previously shown to receive anterograde projections from the DRt. The following discussion on functional considerations of brainstem DRt efferents will focus areas that have not been assigned in previous studies as DRt targets and/or when contradictory conclusions exist among different studies.

Orofacial motor nuclei. The present results demonstrate that the DRt is a major source of afferent input into the orofacial motor nuclei, confirming previous retrograde (Borke et al., 1983; Hinrichsen and Watson, 1983; Travers and Norgren, 1983; Li et al., 1993; Li YQ et al., 1997) and anterograde (Bernard et al., 1990) tracing studies. All the three orofacial motor nuclei Mo5, 7 and 12 receive bilateral projections with ipsilateral prevalence. This same pattern was also obtained after PHA-L administration to the rostral continuation of the DRt, the PCRt (Ter Horst et al., 1991) and in the retrograde studies cited above. Differently, PHA-L injections in the DRt performed by Bernard et al. (1990) resulted in contralateral projections to the Mo5 and 12 and bilateral projections to the 7 , constituting the sole exception to the pattern described.

Orofacial motor behavior results from the complex interaction between jaw, facial and lingual muscles (Fay and Norgren, 1997a,b,c), which are controlled, respectively, by brainstem motoneurons located in the Mo5, 7 and 12 nuclei. There is presently no indication of any large interconnectivity between these motoneurons and thus a reg- 
ulatory role is necessarily attributable to premotor neurons and other hierarchically superior neurons. It is possible that DRt premotor neurons might be involved in the organization of facial expressions and vocalization triggered by noxious stimulation. Firstly, DRt neurons are activated almost exclusively by noxious stimulation (Villanueva et al., 1988). Secondly, double-labeling tracing studies showed that a single DRt neuron can project simultaneously to Mo5/7, Mo5/12 or 7/12 motoneurons (Li et al., 1993; Dauvergne et al., 2001; Popratiloff et al., 2001). Lastly, the DRt receives afferent input from the somatosensory and motor cortices (Almeida et al., 2002; Hattox et al., 2002), adding further complexity to this circuitry.

Endogenous pain control circuitry. Our results on DRt projections to the RVM are largely confirmed by the retrograde work by Hermann et al. (1997), where systematic injections of CTb in the rostral and caudal raphe nucleus and in the GiA resulted in a moderate number of retrogradely labeled neurons in the DRt. No projections to PAG have been demonstrated before, but the efferent projections from the DRt to the PAG are confirmed by our own retrograde control injections of CTb in the PAG. Both the PAG and the RVM are recognized as major players in the pain descending modulatory axis. It is known that electrical stimulation of the PAG can elicit generalized analgesia without loss of any other sensitive or motor function in rats (Reynolds, 1969) and humans (Hosobuchi et al., 1977; Richardson and Akil, 1977; Young et al., 1985). This effect is mediated via RVM, which projects to the spinal cord and modulates spinal nociceptive transmission (see references in Basbaum and Fields, 1984; Jones, 1992). Additionally, besides a pain inhibitory action, the RVM can also facilitate pain (Zhuo and Gebhart, 1997; Porreca et al., 2002). Neural tracing studies indicate that the DRt projects to (present data; Tavares and Lima, 1994; Hermann et al., 1997) and receives projections from (Almeida and Lima, 1997; Almeida et al., 2002) the PAGRVM-dorsal horn circuitry. These anatomical data are per se suggestive of some functional interaction between these areas in pain supraspinal processing (Pertovaara, 2000; Lima and Almeida, 2002; Porreca et al., 2002), even though electrophysiological studies are required to clarify the extension of this reciprocity and its role in pain modulation.

A retrograde study by Luppi et al. (1995) shows that CTb injections restricted to LC core plus pericoerular area resulted in a higher number of cells labeled back in DRt than those restricted to the LC core. Our anterograde data in the present study confirm the existence of DRt projections to the LC and pericoerular area. Additionally, we also demonstrate the existence of DRt efferents to the pontine lateral tegmental cell groups A5 and A7. These data indicate an extensive connectivity between the DRt and the supraspinal antinociceptive noradrenergic system (reviewed by Proudfit, 1988; Berridge and Waterhouse, 2003; Aston-Jones, 2004). In parallel with the spino-PAG-LC (Luppi et al., 1995) and the spino-VLMlat-A5 (Tavares et al., 1996) pathways, DRt projections to LC/pericoeruleus, A5 and $A 7$ represent another possible route through which ascending nociception can reach these noradrenergic areas.

Diencephalon. DRt diencephalic projections were studied in detail after PHA-L administration to the DRt (Villanueva et al., 1998) and, except for some specific areas, no major differences exist between this and the present study with regard to thalamic projections. Furthermore, an exhaustive retrograde study covering a wide range of thalamic areas (Krout et al., 2002) gives a strong support to both anterograde studies. Nonetheless, some exceptions are worth mentioning. With regard to the $\mathrm{Re}$, not all of our BDA injections in the DRt have resulted in labeled terminals in this area and, when they did, only a minor number of terminals were visualized. Similarly, two retrograde works have found few (Krout et al., 2002) or no (McKenna and Vertes, 2004) cells labeled in the DRt after $\mathrm{CTb}$ injections in the Re. Contrastingly, Villanueva's group described a considerable amount of labeled terminals in the $\mathrm{Re}$, especially in its caudal aspect, after PHA injections in the DRt (Villanueva et al., 1998). With regard to the thalamic Rh, we show projections to this nucleus, which is supported by the retrograde (Krout et al., 2002) but is in disagreement with the other anterograde (Villanueva et al., 1998) study.

At the hypothalamic level we have found a number of areas that, with the exception of the LH (Villanueva et al., 1998), are shown here, for the first time, to receive projections from the DRt using anterograde tracing. Previous retrograde studies had already given indications of $\mathrm{DRt}$ projections to the Arc, PVN nuclei and $\mathrm{LH}$, areas implicated in supraspinal pain modulation (Aimone et al., 1988; Yirmiya et al., 1990; Bach, 1997).

Telencephalon. We report here, for the first time, projections from the DRt to the basal ganglia, namely to the SN, MGP and LGP. Additionally, we also report projections to medial/lateral septal areas and, in two cases, projections to the Amy. Injection of the retrograde tracers wheat germ agglutinin-horseradish peroxidase (WGAHRP; Vertes, 1988) and FG (Li JL et al., 1997) in septal areas resulted in contradictory data in respect to the existence of DRt neurons projecting to the septum. In the first report, no labeled neurons were found in the DRt while in the second study a small number of neurons were present in the caudal DRt after FG injection in the VDB/ HDB. Differences between the two tracers and/or in the exact areas to which the tracers were administrated might explain the two apparently contradictory results. In the case of the Amy we have not found retrograde information supporting our data.

From the functional point of view, these areas have been directly or indirectly involved in pain modulation. The basal ganglia, besides their well-known commitment with motor behavior (reviewed by Takakusaki et al., 2004) has also been associated with pain modulation (reviewed by Chudler and Dong, 1995). In the case of septal nuclei, two reports have shown that changes in the hippocampal cell activity induced by noxious stimulation are impeded by VDB lesions (Khanna, 1997; Khanna and Zheng, 1999). Finally, the Amy has a well-known role as a higher center 
in the regulation of brainstem endogenous pain control system (Neugebauer et al., 2004) and is the main regulator of the emotional component of pain. Taking into account these data from other groups, the anatomical data reported here and the well-known role of DRt in pain modulation (Villanueva et al., 1996; Lima and Almeida, 2002), it is possible to speculate that peripheral noxious information may be relayed, at least in part, in the DRt before transmission to forebrain centers. What is the exact role of these circuits in pain modulation (or in other functions) and how these areas interact in order to produce an output (ultimately a behavior or a sensation) are yet to be found.

Acknowledgments-This study was supported by Project POCTII NSE/46399/2002 from Fundação para a Ciência e Tecnologia (FCT) and FEDER, and by Grünenthal Foundation (Portugal).

\section{REFERENCES}

Aimone LD, Bauer CA, Gebhart GF (1988) Brain-stem relays mediating stimulation-produced antinociception from the lateral hypothalamus in the rat. J Neurosci 8:2652-2663.

Almeida A, Cobos A, Tavares I, Lima D (2002) Brain afferents to the medullary dorsal reticular nucleus: a retrograde and anterograde tracing study in the rat. Eur J Neurosci 16:81-95.

Almeida A, Lima D (1997) Activation by cutaneous or visceral noxious stimulation of spinal neurons projecting to the medullary dorsal reticular nucleus in the rat: a c-fos study. Eur $\mathrm{J}$ Neurosci 9: 686-695.

Almeida A, Storkson R, Lima D, Hole K, Tjolsen A (1999) The medullary dorsal reticular nucleus facilitates pain behaviour induced by formalin in the rat. Eur J Neurosci 11:110-122.

Almeida A, Tavares I, Lima D (1995) Projection sites of superficial or deep dorsal horn in the dorsal reticular nucleus. Neuroreport 6:1245-1248.

Almeida A, Tavares I, Lima D (2000) Reciprocal connections between the medullary dorsal reticular nucleus and the spinal dorsal horn in the rat. Eur J Pain 4:373-387.

Almeida A, Tavares I, Lima D, Coimbra A (1993) Descending projections from the medullary dorsal reticular nucleus make synaptic contacts with spinal cord lamina I cells projecting to that nucleus: an electron microscopic tracer study in the rat. Neuroscience 55:1093-1106.

Almeida A, Tjolsen A, Lima D, Coimbra A, Hole K (1996) The medullary dorsal reticular nucleus facilitates acute nociception in the rat. Brain Res Bull 39:7-15.

Arends JJ, Wild JM, Zeigler HP (1988) Projections of the nucleus of the tractus solitarius in the pigeon (Columba livia). J Comp Neurol 278:405-429.

Aston-Jones G (2004) Locus coeruleus, A5 and A7 noradrenergic cell groups. In: The rat nervous system (Paxinos G, ed), pp 259-294. London: Elsevier.

Babic T, Roder S, Ciriello J (2004) Direct projections from caudal ventrolateral medullary depressor sites to the subfornical organ. Brain Res 1003:113-121.

Bach FW (1997) Beta-endorphin in the brain. A role in nociception. Acta Anaesthesiol Scand 41:133-140.

Basbaum Al, Fields HL (1984) Endogenous pain control systems: brainstem spinal pathways and endorphin circuitry. Annu Rev Neurosci 7:309-338.

Bernard JF, Villanueva L, Carroue J, Le Bars D (1990) Efferent projections from the subnucleus reticularis dorsalis (SRD): a Phaseolus vulgaris leucoagglutinin study in the rat. Neurosci Lett 116: 257-262.
Berridge CW, Waterhouse BD (2003) The locus coeruleus-noradrenergic system: modulation of behavioral state and state-dependent cognitive processes. Brain Res Brain Res Rev 42:33-84.

Bobillier P, Seguin S, Petitjean F, Salvert D, Touret M, Jouvet M (1976) The raphe nuclei of the cat brain stem: a topographical atlas of their efferent projections as revealed by autoradiography. Brain Res 113:449-486.

Bodnar RJ (2000) Supraspinal circuitry mediating opioid antinociception: antagonist and synergy studies in multiple sites. J Biomed Sci 7: 181-194.

Borke RC, Nau ME, Ringler RL Jr (1983) Brain stem afferents of hypoglossal neurons in the rat. Brain Res 269:47-55.

Bouhassira D, Villanueva L, Bing Z, le Bars D (1992) Involvement of the subnucleus reticularis dorsalis in diffuse noxious inhibitory controls in the rat. Brain Res 595:353-357.

Cameron AA, Khan IA, Westlund KN, Cliffer KD, Willis WD (1995a) The efferent projections of the periaqueductal gray in the rat: a Phaseolus vulgaris-leucoagglutinin study. I. Ascending projections. J Comp Neurol 351:568-584.

Cameron AA, Khan IA, Westlund KN, Willis WD (1995b) The efferent projections of the periaqueductal gray in the rat: a Phaseolus vulgaris-leucoagglutinin study. II. Descending projections. J Comp Neurol 351:585-601.

Chudler EH, Dong WK (1995) The role of the basal ganglia in nociception and pain. Pain 60:3-38.

Cliffer KD, Burnstein R, Giesler GL (1991) Distributions of spinothalamic, spinohypothalamic, and spinotelencephalic fibers revealed by anterograde transport of PHA-L in rats. J Neurosci 11:852-868.

Cobos A, Lima D, Almeida A, Tavares I (2003) Brain afferents to the lateral caudal ventrolateral medulla: a retrograde and anterograde tracing study in the rat. Neuroscience 120:485-498.

Dauvergne C, Pinganaud G, Buisseret P, Buisseret-Delmas C, ZerariMailly F (2001) Reticular premotor neurons projecting to both facial and hypoglossal nuclei receive trigeminal afferents in rats. Neurosci Lett 311:109-112.

Donovick PJ (1974) A metachromatic stain for neural tissue. Stain Technol 49:49-51.

Dugast C, Almeida A, Lima D (2003) The medullary dorsal reticular nucleus enhances the responsiveness of spinal nociceptive neurons to peripheral stimulation in the rat. Eur $\mathrm{J}$ Neurosci 18: 580-588.

Fay RA, Norgren R (1997a) Identification of rat brainstem multisynaptic connections to the oral motor nuclei in the rat using pseudorabies virus. II. Facial muscle motor systems. Brain Res Brain Res Rev 25:276-290.

Fay RA, Norgren R (1997b) Identification of rat brainstem multisynaptic connections to the oral motor nuclei using pseudorabies virus. I. Masticatory muscle motor systems. Brain Res Brain Res Rev 25:255-275.

Fay RA, Norgren R (1997c) Identification of rat brainstem multisynaptic connections to the oral motor nuclei using pseudorabies virus. III. Lingual muscle motor systems. Brain Res Brain Res Rev 25: 291-311.

Gauriau C, Bernard JF (2004) A comparative reappraisal of projections from the superficial laminae of the dorsal horn in the rat: the forebrain. J Comp Neurol 468:24-56.

Gebhart GF (2004) Descending modulation of pain. Neurosci Biobehav Rev 27:729-737.

Hattox AM, Priest CA, Keller A (2002) Functional circuitry involved in the regulation of whisker movements. J Comp Neurol 442: 266-276.

Herbert H, Klepper A, Ostwald J (1997) Afferent and efferent connections of the ventrolateral tegmental area in the rat. Anat Embryol (Berl) 196:235-259.

Hermann DM, Luppi PH, Peyron C, Hinckel P, Jouvet M (1997) Afferent projections to the rat nuclei raphe magnus, raphe pallidus and reticularis gigantocellularis pars alpha demonstrated by ionto- 
phoretic application of choleratoxin (subunit b). J Chem Neuroanat 13:1-21.

Hinrichsen CF, Watson CD (1983) Brain stem projections to the facial nucleus of the rat. Brain Behav Evol 22:153-163.

Hosobuchi Y, Adams JE, Linchitz R (1977) Pain relief by electrical stimulation of the central gray matter in humans and its reversal by naloxone. Science 197:183-186.

Jones BE, Yang TZ (1985) The efferent projections from the reticular formation and the locus coeruleus studied by anterograde and retrograde axonal transport in the rat. J Comp Neurol 242:56-92.

Jones SL (1991) Descending noradrenergic influences on pain. Prog Brain Res 88:381-394.

Jones SL (1992) Descending control of nociception. In: The initial processing of pain and its descending control: spinal and trigeminal systems, Vol. 12 (Light AR, ed), pp 203-295. Basel, Switzerland: Karger.

Joseph SA, Micheal GJ (1988) Efferent ACTH-IR opiocortin projections from nucleus tractus solitarius: a hypothalamic deafferentation study. Peptides 9:193-201.

Khanna S (1997) Dorsal hippocampus field CA1 pyramidal cell responses to a persistent versus an acute nociceptive stimulus and their septal modulation. Neuroscience 77:713-721.

Khanna S, Zheng F (1999) Morphine reversed formalin-induced CA1 pyramidal cell suppression via an effect on septohippocampal neural processing. Neuroscience 89:61-71.

Köbbert C, Apps R, Bechmann I, Lanciego JL, Mey J, Thanos S (2000) Current concepts in neuroanatomical tracing. Prog Neurobiol 62: 327-351.

Koutcherov Y, Huang X-F, Halliday G, Paxinos G (2004) Organization of human brain stem nuclei. In: The human nervous system (Paxinos G, Mai JK, eds), pp 267-320. London: Elsevier-Academic Press.

Krout KE, Belzer RE, Loewy AD (2002) Brainstem projections to midline and intralaminar thalamic nuclei of the rat. J Comp Neurol 448:53-101.

Leite-Almeida H, Almeida A (2004) Brain projections from the medullary dorsal reticular nucleus (DRt) in the rat: an anterograde tracing study. Proceedings of the FENS Forum 2004, Lisbon, 2, p A192.118.

Li JL, Kaneko T, Shigemoto R, Mizuno N (1997) Distribution of trigeminohypothalamic and spinohypothalamic tract neurons displaying substance $P$ receptor-like immunoreactivity in the rat. J Comp Neurol 378:508-521.

Li YQ, Takada M, Kaneko T, Mizuno N (1997) Distribution of GABAergic and glycinergic premotor neurons projecting to the facial and hypoglossal nuclei in the rat. J Comp Neurol 378:283-294.

Li YQ, Takada M, Mizuno N (1993) Premotor neurons projecting simultaneously to two orofacial motor nuclei by sending their branched axons. A study with a fluorescent retrograde doublelabeling technique in the rat. Neurosci Lett 152:29-32.

Lima D (1990) A spinomedullary projection terminating in the dorsal reticular nucleus of the rat. Neuroscience 34:577-589.

Lima D, Almeida A (2002) The medullary dorsal reticular nucleus as a pronociceptive centre of the pain control system. Prog Neurobiol 66:81-108

Lima D, Coimbra A (1988) The spinothalamic system of the rat: structural types of retrogradely labelled neurons in the marginal zone (lamina I). Neuroscience 27:215-230.

Luppi PH, Aston-Jones G, Akaoka H, Chouvet G, Jouvet M (1995) Afferent projections to the rat locus coeruleus demonstrated by retrograde and anterograde tracing with cholera-toxin B subunit and Phaseolus vulgaris leucoagglutinin. Neuroscience 65: $119-160$.

Mason P (2001) Contributions of the medullary raphe and ventromedial reticular region to pain modulation and other homeostatic functions. Annu Rev Neurosci 24:737-777.

McKenna JT, Vertes RP (2004) Afferent projections to nucleus reuniens of the thalamus. J Comp Neurol 480:115-142.
Menetrey D, Basbaum Al (1987) Spinal and trigeminal projections to the nucleus of the solitary tract: a possible substrate for somatovisceral and viscerovisceral reflex activation. J Comp Neurol 255: $439-450$.

Millan MJ (2002) Descending control of pain. Prog Neurobiol 66: 355-474.

Monconduit L, Desbois C, Villanueva L (2002) The integrative role of the rat medullary subnucleus reticularis dorsalis in nociception. Eur J Neurosci 16:937-944.

Neto FL, Schadrack J, Ableitner A, Castro-Lopes JM, Bartenstein P, Zieglgansberger W, Tolle TR (1999) Supraspinal metabolic activity changes in the rat during adjuvant monoarthritis. Neuroscience 94:607-621.

Neugebauer V, Li W, Bird GC, Han JS (2004) The amygdala and persistent pain. Neuroscientist 10:221-234.

Newman DB (1985) Distinguishing rat brainstem reticulospinal nuclei by their neuronal morphology. I. Medullary nuclei. J Hirnforsch 26:187-226.

Novikov LN (2001) Labeling of central projections of primary afferents in adult rats: a comparison between biotinylated dextran amine, neurobiotin and Phaseolus vulgaris-leucoagglutinin. J Neurosci Methods 112:145-154.

Paxinos G, Watson C (1998) The rat brain in stereotaxic coordinates. New York: Academic Press.

Paxinos G, Watson C (2005) The rat brain in stereotaxic coordinates. New York: Academic Press.

Pertovaara A (2000) Plasticity in descending pain modulatory systems. Progress in brain research, pp 231-242. Elsevier.

Popratiloff AS, Streppel M, Gruart A, Guntinas-Lichius O, Angelov DN, Stennert E, Delgado-Garcia JM, Neiss WF (2001) Hypoglossal and reticular interneurons involved in oro-facial coordination in the rat. J Comp Neurol 433:364-379.

Porreca F, Ossipov MH, Gebhart GF (2002) Chronic pain and medullary descending facilitation. Trends Neurosci 25:319-325.

Porro CA (2003) Functional imaging and pain: behavior, perception, and modulation. Neuroscientist 9:354-369.

Proudfit HK (1988) Pharmacologic evidence for the modulation of nociception by noradrenergic neurons. Prog Brain Res 77:357-370.

Raboisson P, Dallel R, Bernard JF, Le Bars D, Villanueva L (1996) Organization of efferent projections from the spinal cervical enlargement to the medullary subnucleus reticularis dorsalis and the adjacent cuneate nucleus: a PHA-L study in the rat. J Comp Neurol 367:503-517.

Randich A, Roose MG, Gebhart GF (1988) Characterization of antinociception produced by glutamate microinjection in the nucleus tractus solitarius and the nucleus reticularis ventralis. J Neurosci 8:4675-4684.

Reynolds DV (1969) Surgery in the rat during electrical analgesia induced by focal brain stimulation. Science 164:444-445.

Richardson DE, Akil H (1977) Pain reduction by electrical brain stimulation in man. Part 1: Acute administration in periaqueductal and periventricular sites. J Neurosurg 47:178-183.

Takakusaki K, Saitoh K, Harada H, Kashiwayanagi M (2004) Role of basal ganglia-brainstem pathways in the control of motor behaviors. Neurosci Res 50:137-151.

Tavares I, Lima D (1994) Descending projections from the caudal medulla oblongata to the superficial or deep dorsal horn of the rat spinal cord. Exp Brain Res 99:455-463.

Tavares I, Lima D (2002) The caudal ventrolateral medulla as an important inhibitory modulator of pain transmission in the spinal cord. J Pain 3:337-346.

Tavares I, Lima D, Coimbra A (1996) The ventrolateral medulla of the rat is connected with the spinal cord dorsal horn by an indirect descending pathway relayed in the A5 noradrenergic cell group. J Comp Neurol 374:84-95.

Ter Horst GJ, Copray JC, Liem RS, Van Willigen JD (1991) Projections from the rostral parvocellular reticular formation to pontine and 
medullary nuclei in the rat: involvement in autonomic regulation and orofacial motor control. Neuroscience 40:735-758.

Travers JB, Norgren R (1983) Afferent projections to the oral motor nuclei in the rat. J Comp Neurol 220:280-298.

Valverde $F$ (1962) Reticular formation of the albino rat's brain stem cytoarchitecture and corticofugal connections. J Comp Neurol 119: 25-53.

Vertes RP (1988) Brainstem afferents to the basal forebrain in the rat. Neuroscience 24:907-935.

Villanueva L, Bernard JF, Le Bars D (1995) Distribution of spinal cord projections from the medullary subnucleus reticularis dorsalis and the adjacent cuneate nucleus: a Phaseolus vulgaris-leucoagglutinin study in the rat. J Comp Neurol 352:11-32.

Villanueva L, Bing Z, Bouhassira D, Le Bars D (1989) Encoding of electrical, thermal, and mechanical noxious stimuli by subnucleus reticularis dorsalis neurons in the rat medulla. J Neurophysiol 61: 391-402.

Villanueva L, Bouhassira D, Bing Z, Le Bars D (1988) Convergence of heterotopic nociceptive information onto subnucleus reticularis dorsalis neurons in the rat medulla. J Neurophysiol 60:980-1009.

Villanueva L, Bouhassira D, Le Bars D (1996) The medullary subnucleus reticularis dorsalis (SRD) as a key link in both the transmission and modulation of pain signals. Pain 67:231-240.

Villanueva L, Cliffer KD, Sorkin LS, Le Bars D, Willis WD Jr (1990) Convergence of heterotopic nociceptive information onto neurons of caudal medullary reticular formation in monkey (Macaca fascicularis). J Neurophysiol 63:1118-1127.

Villanueva L, Desbois C, Le Bars D, Bernard JF (1998) Organization of diencephalic projections from the medullary subnucleus reticularis dorsalis and the adjacent cuneate nucleus: a retrograde and anterograde tracer study in the rat. J Comp Neurol 390:133-160.

Wang CC, Willis WD, Westlund KN (1999) Ascending projections from the area around the spinal cord central canal: A Phaseolus vulgaris leucoagglutinin study in rats. J Comp Neurol 415:341-367.

Wiertelak EP, Roemer B, Maier SF, Watkins LR (1997) Comparison of the effects of nucleus tractus solitarius and ventral medial medulla lesions on illness-induced and subcutaneous formalin-induced hyperalgesias. Brain Res 748:143-150.

Yirmiya R, Ben-Eliyahu S, Shavit Y, Marek P, Liebeskind JC (1990) Stimulation of the hypothalamic paraventricular nucleus produces analgesia not mediated by vasopressin or endogenous opioids. Brain Res 537:169-174.

Young RF, Kroening R, Fulton W, Feldman RA, Chambi I (1985) Electrical stimulation of the brain in treatment of chronic pain. Experience over 5 years. J Neurosurg 62:389-396.

Zhuo M, Gebhart GF (1997) Biphasic modulation of spinal nociceptive transmission from the medullary raphe nuclei in the rat. J Neurophysiol 78:746-758.

(Accepted 10 February 2006) (Available online 23 March 2006) 